\title{
Diversidad de insectos acuáticos en quebradas impactadas por agricultura y minería, Caldas, Colombia
}

\author{
Sebastián Villada-Bedoya ${ }^{1,2 *}$, Fabiola Ospina-Bautista ${ }^{1,3}$, Lucimar G-Dias $^{2}$
}

\& Jaime Vicente Estévez Varón ${ }^{1}$

1. Departamento de Ciencias Biológicas, Facultad de Ciencias Exactas y Naturales. Grupo de investigación en Ecosistemas Tropicales, Universidad de Caldas, Calle 65 \# 26-10, Manizales, Colombia. Apartado Aéreo 275; escarasebas@gmail.com, jaime.estevez@ucaldas.edu.co

2. Departamento de Ciencias Biológicas, Facultad de Ciencias Exactas y Naturales. Grupo de investigación en Biodiversidad y Recursos Naturales (Bionat), Universidad de Caldas, Calle 65 \# 26-10, Manizales, Colombia. Apartado Aéreo 275; lucimar.dias@ucaldas.edu.co

3. Laboratorio de Ecología acuática y Zoología (LAZOEA), Universidad de los Andes. Bogotá, Colombia; fabiola.ospina@ucaldas.edu.co

* Correspondencia

Recibido 23-II-2017. Corregido 11-VII-2017. Aceptado 10-VIII-2017.

\begin{abstract}
Insect diversity in streams impacted by agriculture and mining, Caldas, Colombia. Freshwater ecosystems are under severe anthropic pressure (e.g. agriculture and mining), which generates adverse effects on the diversity of aquatic insects. We evaluated the insect community in streams impacted by agriculture and mining, and determined how these activities affected the community. We studied six streams in Caldas (Colombia), two with mining and two with agriculture impact. We collected the insects using a Surber net with three replicates per substrate (fine sediment, stones, and litter). In total, 12502 aquatic insects were collected. The most representative genera were Baetodes, Simulium, and Cricotopus (57.7\%). Streams with agricultural activity had lower aquatic insects diversity than mining impact $\left(\mathrm{F}_{2,309}=21.82, \mathrm{p}=0.001\right)$. The agriculture areas presented a structure dominated by Simulium and Cricotopus (Diptera, pollution-tolerant morphospecies). Baetodes dominated in stones substrate, Cricotopus in sediment, and Simulium and Cricotopus dominated in litter $\left(\mathrm{F}_{2,309}=2.84, \mathrm{p}=0.001\right)$. Therefore, both factors (impact and substrate type) are being important in determining the diversity of aquatic insects. Our results suggested that agricultural activity has a higher impact on the diversity of aquatic insects, which may be associated with the loss of riparian vegetation. Rev. Biol. Trop. 65 (4): 1635-1659. Epub 2017 December 01.
\end{abstract}

Key words: aquatic ecosystems, Colombian Andes, anthropic disturbance, benthic insects.

Una de las principales causas de pérdida de la biodiversidad en las últimas décadas han sido las actividades de origen antrópico (Fahrig, 2003), que alteran de forma directa los ecosistemas dulceacuícolas (Wiens, 2002; Nilsson, Reidy, Dynesius, \& Revenga, 2005; Etter, McAlpine, Wilson, Phinn, \& Possingham, 2006), que a su vez, son considerados uno de los recursos naturales más importantes en términos de su valor biológico (Jonsson, Malmqvist, \& Hoffsten, 2001). Estos ecosistemas sustentan una alta diversidad, donde se destacan los insectos acuáticos, que son un componente importante de la fauna dulceacuícola y han sido usados ampliamente como grupo indicador ecológico de perturbación antrópica (Reece \& Richardson, 2000; Merritt, Cummins, \& Berg, 2008; Guevara, Godoy, Boeckx, Jara, \& Oyarzún, 2009), debido a que las alteraciones causan cambios en la estructura y composición de dichas comunidades (Barbour, Gerritsen, Snyder, \& Stribling, 1999; Wiens 2002).

La agricultura y la minería están entre las principales actividades que impactan 
negativamente la estructura del suelo, los recursos hídricos y la biodiversidad a nivel mundial (Stanford et al., 1996; Wiens, 2002; Nilsson et al., 2005). Esto también ha sucedido en los Andes (Etter et al., 2006; Defensoría del pueblo Colombia, 2010), debido a la pérdida de la vegetación ribereña, la erosión y el uso de sustancias contaminantes (Ramírez, Pringle, \& Wantzen, 2008; Brosse, Grenouillet, Gevrey, Khazraie, \& Tudesque, 2011). Estas actividades tienen efectos negativos sobre las cadenas tróficas (Canton \& Ward, 1981; Boudou, Maury-Brachet, Coquery, Durrieu, \& Cossa, 2005; Pond, Passmore, Borsuk, Reynolds, \& Rose, 2008) y el funcionamiento de los ecosistemas (Barbour et al., 1999; Ward, Tockner, \& Schiemer, 1999). En las últimas cinco décadas, la agricultura en Colombia ha tenido un crecimiento acelerado, debido a la intensificación de la producción y a la expansión de los cultivos (Etter et al., 2006), y se ha documentado que es una de las actividades antrópicas que más altera las dinámicas de los ecosistemas acuáticos (Maloney \& Weller, 2011; Piggott, Lange, Townsend, \& Matthaei, 2012; Chará-Serna, Chará, Giraldo, Zúñiga, \& Allan, 2015). Por otro lado, la minería es una de las actividades que más impacta los ecosistemas lóticos, ya que estos actúan como receptores de los desechos provenientes de la explotación y extracción de minerales (Bernhardt \& Palmer, 2011; Brosse et al. 2011).

Los efectos de la perturbación antrópica (i.e. agricultura y minería) sobre la diversidad biológica han sido ampliamente estudiados, sin embargo, aún no existe un consenso acerca de la respuesta de la biodiversidad a la perturbación (Huston, 1994; Lal, 1998; Mackey \& Currie, 2001; Bann, Alkemade, \& Koellner, 2013; Newbold et al. 2015). La evaluación de la diversidad de insectos acuáticos en ecosistemas dulceacuícolas, ha sido un tema de principal interés (Chará-Serna, Chará, Zúñiga, Pedraza, \& Giraldo, 2010; Rodríguez-Barrios, Ospina-Torres, \& Turizo-Correa, 2011; González, Ramírez, Meza-S, \& G-Dias, 2012; Meza-S, Rubio-M, G-Dias, \& Walteros, 2012; Chará-Serna et al., 2015) para el entendimiento de las dinámicas ecológicas al interior de las quebrabas tropicales, debido a que son fuente primaria dentro de la cadena trófica, y participan activamente en la descomposición de la materia orgánica y el ciclo de nutrientes (Reece \& Richardson, 2000; Guevara et al., 2009).

Establecer los efectos de la actividad agrícola y minera sobre la diversidad de insectos acuáticos y su potencial impacto sobre los ecosistemas dulceacuícolas, es relevante dado el incremento de estas actividades en Latinoamérica (Harvey et al., 2008; Brosse et al., 2011; Armenteras \& Rodríguez, 2014; Chará-Serna et al., 2015). En la actualidad, no se conocen con certeza los efectos sobre las comunidades asociadas a ecosistemas acuáticos inmersos en matrices con presencia de actividades agrícolas y mineras (Pond et al., 2008; Hepp, Milesi, Biasi, \& Restello, 2010; Piggott et al., 2012; De Jonge, Tipping, Lofts, Bervoets, \& Blust, 2013; Mondal \& Jana, 2015) en quebradas de los Andes tropicales, las cuales se caracterizan por ser pequeñas, correntosas y propensas a la erosión, debido a las fuertes lluvias (Chará-Serna et al., 2015). Sin embargo, se ha documentado que ambas actividades causan contaminación agroquímica, incremento de la carga orgánica, aumento de la sedimentación y mineralización en los ecosistemas acuáticos (Coutinho, Noellemeyyer, Jobbagy, Jonathan, \& Paruelo, 2009; Hepp et al., 2010; Palmer et al., 2010).

La agricultura y la minería como impactos, incrementan la pérdida de la vegetación ribereña (protección natural contra la erosión) y a su vez la entrada de residuos orgánicos, nutrientes, metales (Allan, 2004; Milesi, Biasi, Restello, \& Hepp, 2008), sedimentos y otros contaminantes a las quebradas (Bjerklie \& LaPerriere, 1985; Nessimian et al., 2008). Todos estos factores pueden incrementar el $\mathrm{pH}$, la conductividad eléctrica y los sólidos disueltos totales (Palmer et al., 2010) al interior de los ríos y quebradas. Por otro lado, el tiempo de uso del suelo y la ubicación con respecto al impacto en cada localidad, hace que la perturbación tenga un nivel de impacto variable (Wood \& Armitage, 1997).

En particular, este estudio evaluó la diversidad de insectos acuáticos expuestos 
a perturbación de tipo agrícola y minera en quebradas de los Andes colombianos. Las actividades de tipo agrícola y minero alteran negativamente los ecosistemas acuáticos, las cuales generan cambios estructurales (en la vegetación ribereña), físicos y químicos de los ecosistemas, y por consiguiente una disminución de la diversidad de insectos acuáticos (Allan, 2004; Harvey et al., 2008; Yoshimura, 2012; Chará-Serna et al., 2015). Por lo tanto, se predice que la riqueza, abundancia y composición de la comunidad de insectos se verán alteradas por la actividad minera, debido al uso de metales pesados (mercurio y cianuro) usados para la extracción de oro y el tiempo de exposición de los organismos a estas sustancias. Por otro lado, la agricultura puede afectar la comunidad debido a la historia de uso del suelo ( $\sim 90$ años) en la zona de estudio y el uso de herbicidas que causa cambios estructurales en el ecosistema (pérdida de vegetación ribereña) y puede estar modulando la respuesta de los insectos acuáticos en cada quebrada evaluada. Además, es posible que los efectos de la minería y la agricultura sean dependientes de las características fisicoquímicas, hidrológicas, estructurales del hábitat y la ubicación del impacto, que determinarán la presencia de las morfoespecies tolerantes a estos cambios.

\section{MATERIALES Y MÉTODOS}

Área de estudio: El estudio se llevó a cabo en dos localidades del municipio de Villamaría (Gallinazo: 459'22” N - 75²5'58” W y Llanitos: 5'1'42.1" $\mathrm{N}-75^{\circ} 31$ '10.9" W), ubicadas sobre la vertiente occidental de la cordillera Central de los Andes colombianos en el departamento de Caldas y con una distancia entre ellas mayor a $5 \mathrm{~km}$. El rango altitudinal de los sitios de estudio se encuentra entre los 1720 y los 2305 m, y de acuerdo a Holdridge (1982) corresponde a una zona de bosque húmedo premontano (bmh-PM), con una temperatura que oscila entre 15 y $20^{\circ} \mathrm{C}$. Cada localidad de muestreo presenta un tipo de impacto, la primera con impacto minero (Gallinazo), donde se realiza extracción de oro y donde se utiliza mercurio $(\mathrm{Hg})$ para la separación del mineral, la explotación se hace a pequeña escala desde hace aproximadamente 50 años (com. pers.). La segunda con impacto agrícola (Llanitos), donde predomina el cultivo de hortalizas con manejo tradicional, este incluye el uso de pesticidas (e.g. Glifosato), fertilizantes de síntesis química y prácticas que incluyen la remoción de suelo (Nicholls, 2008), esta actividad se realiza continuamente desde hace $\sim 90$ años (com. pers.). En cada localidad se seleccionó una zona de referencia (quebradas sin ningún tipo de impacto). Se eligieron tres quebradas en cada localidad, en la localidad Gallinazo las quebradas Romerales (referencia), California y Toldafría (impacto minero), y en la localidad Llanitos las quebradas El Establo (referencia), Don Alonso y Don Fernando (impacto agrícola), todas las quebradas hacen parte de la cuenca alta del río Chinchiná (Fig. 1).

\section{Zona impactada por minería (localidad Gallinazo)}

Quebrada Romerales: Estación 1 (E1, ver Anexo 1), la cual corresponde a la zona de referencia $\left(4^{\circ} 59^{\prime} 22^{\prime \prime} \mathrm{N}-75^{\circ} 25^{\prime} 58^{\prime \prime} \mathrm{W}\right)$ (Fig. 1), está ubicada a $2305 \mathrm{~m}$ de altitud, presenta un ancho de $\sim 6.28 \mathrm{~m}$ y una profundidad de $\sim 17.27 \mathrm{~cm}$, con un caudal de $0.77 \mathrm{~m}^{3} / \mathrm{s}$, predomina el sustrato roca, se presenta bastante acumulación de hojarasca en las orillas y se presentan pequeños remansos con presencia de sedimento fino. Tiene una franja de vegetación ribereña de $\sim 20 \mathrm{~m}$ y un dosel que alcanza los $30 \mathrm{~m}$. Entre la vegetación presente en el sitio de muestreo se encuentran especies arbóreas de las familias Rubiaceae (Guettarda cf. crispiflora), Chloranthaceae (Hedyosmun bonplandianum), Cunoniaceae (Weinmannia pubescens), Araliaceae (Oreopanax sp.) y Staphyleaceae (Turpinia occidentalis). La vegetación está dominada por el estrato arbustivo con una altura menor a $5 \mathrm{~m}$, donde dominan las familias Melastomataceae (Miconia cf. theaezans), Asteraceae (Elaphandra quinquenervis, Braccharis latifolia) y Acanthaceae (Aphelandra acanthus) y hierbas terrestres. Los géneros 

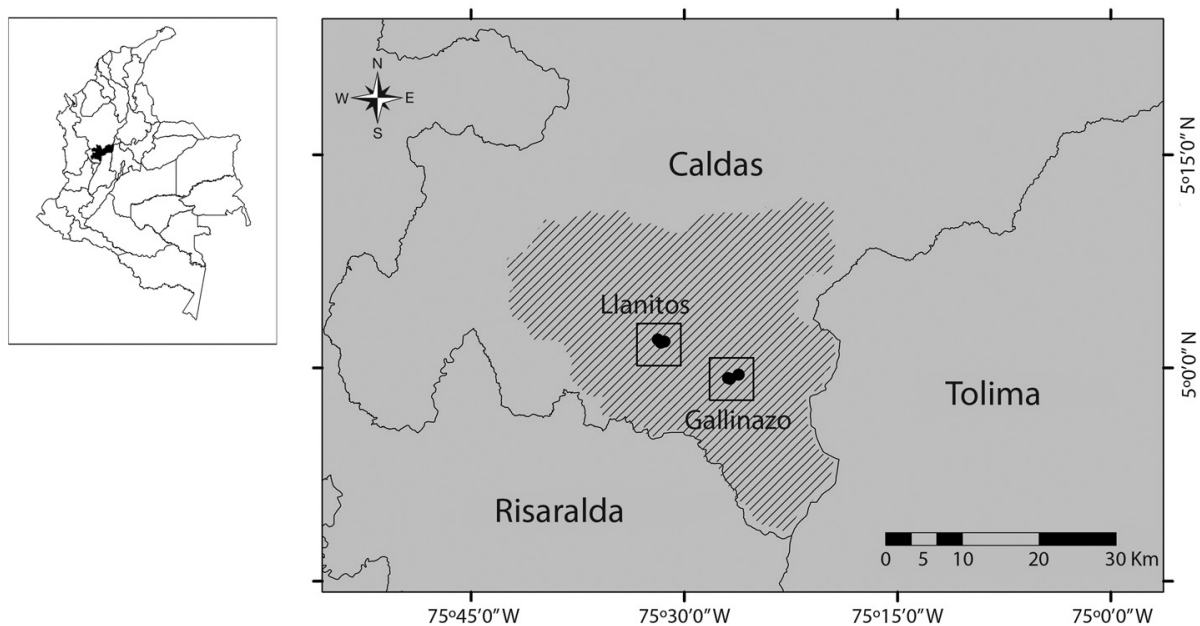

Sitios de muestreo

Cuencia río Chinchiná

- Red hídrica

Uso del suelo

Bosque

Zonas agropecuarias

otros

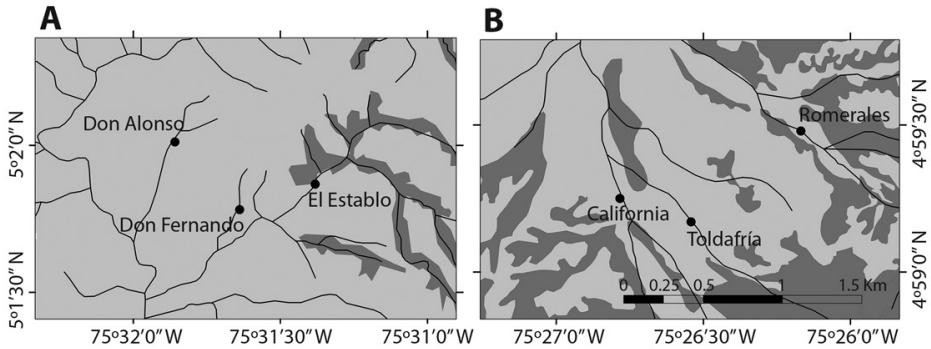

Fig. 1. Área de estudio en el municipio de Villamaria, Caldas. A. Llanitos: zona agrícola y B. Gallinazo: zona minera. Se muestra la ubicación de las quebradas (estaciones de muestreo) en cada zona.

Fig. 1. The study site in the municipality of Villamaría, Caldas. A. Llanitos: agriculture zone and B. Gallinazo: mining zone. The location of streams (sampling stations) shown in each study site.

con mayor riqueza de especies pertenecen a las familias Urticaceae (Pilea), Solanaceae (Solanum), Rosaceae (Rubus) y Melastomatacea (Miconia). En total se registraron 328 individuos en un área total de $2500 \mathrm{~m}^{2}$, de los cuales 41 corresponden a árboles con una altura mayor a $1.3 \mathrm{~m}$ y DAP mayor a $5 \mathrm{~cm}$ (Bedoya \& Orozco, 2015).

Quebrada California: Estación 2 (E2, ver Anexo 1) ubicada a una altitud de 2260 $\mathrm{m}\left(04^{\circ} 59^{\prime} 5^{\prime} \mathrm{N}-75^{\circ} 26^{\prime} 35^{\prime \prime} \mathrm{W}\right)$ (Fig. 1), con un ancho de $\sim 4.67 \mathrm{~m}$ y una profundidad de $22.01 \mathrm{~cm}$, presenta un caudal de $0.71 \mathrm{~m}^{3} / \mathrm{s}$, predomina el sustrato roca, con presencia de grandes bloques de roca, se presenta bastante acumulación de hojarasca en las orillas, el sedimento fino se localiza en las orillas y en pequeños remansos. Corresponde a la primera quebrada con evidencia de impacto minero, una orilla presenta vegetación arbustiva y algunas palmas (Arecaceae) y la otra con zonas de pastos. El terreno presenta pendientes pronunciadas, con vegetación secundaria dominada por las familias Asteraceae, Melastomataceae y Solanaceae. Se registraron 292 individuos en total en un área de $2500 \mathrm{~m}^{2}$, donde 44 corresponden a árboles con una altura mayor a $1.3 \mathrm{~m}$ y DAP mayor a $5 \mathrm{~cm}$; la vegetación está dominada por el estrato arbustivo con una altura menor a $5 \mathrm{~m}$, donde predominan las especies: B. latifolia (Asteraceae), Miconia cf. superposita (Melastomataceae) y Morella pubescens (Myricaceae), con presencia de helechos arbóreos de las familias Dicksoniaceae (Dicksonia sp.) y Cyatheaceae (Sphaeropteris quindiuensis). La vegetación presenta un dosel cercano a los 15 m (Bedoya \& Orozco, 2015). 
Quebrada Toldafría: Estación 3 (E3, ver Anexo 1), corresponde a la segunda quebrada impactada por minería $\left(4^{\circ} 59^{\prime} 08^{\prime \prime} \mathrm{N}\right.$ - 75'26’43” W) a una altitud de $2275 \mathrm{~m}$ (Fig. 1), con un ancho de $\sim 5.12 \mathrm{~m}$ y una profundidad de $\sim 13.49 \mathrm{~cm}$; presenta un caudal de $0.38 \mathrm{~m}^{3} / \mathrm{s}$, predomina el sustrato roca, seguido de sedimento fino, el sustrato hojarasca es menor a $30 \%$ y se presenta en las orillas. Entre la vegetación dominante en esta estación se encuentran las familias Melastomataceae (Leandra subseriata, Miconia smaragdina, $M$. cf. theaezans), Asteraceae (B. latifolia, Erato vulcanica), Myricaceae (M. pubescens) y Solanaceae (Solanum cf. phaeophyllum). La quebrada Toldafría presenta una vegetación dominada por el estrato arbustivo con altura menor a $5 \mathrm{~m}$, similar a la presente en la quebrada California, con un total de 292 individuos registrados en un área de $2500 \mathrm{~m}^{2}$, de los cuales 28 corresponden a árboles con una altura mayor a $1.3 \mathrm{~m}$ y DAP mayor a $5 \mathrm{~cm}$. El dosel no supera los $14 \mathrm{~m}$ y hay presencia de zonas de pastoreo en las orillas de la quebrada (Bedoya \& Orozco, 2015).

\section{Zona impactada por agricultura (localidad Llanitos)}

Quebrada El Establo: Estación 4 (E4, ver Anexo 1), la cual corresponde a la zona de referencia $\left(05^{\circ} 1^{\prime} 42.1^{\prime \prime} \mathrm{N}-75^{\circ} 31\right.$ ' $10.9^{\prime \prime}$ W) (Fig. 1), tiene un ancho de $\sim 1.82 \mathrm{~m}$ y una profundidad de $\sim 7.59 \mathrm{~cm}$, con un caudal de $0.77 \mathrm{~m}^{3} / \mathrm{s}$, predomina el sustrato roca $\mathrm{y}$ material vegetal en descomposición (troncos) con presencia de hojarasca superior al $30 \%$, también se presentan pozos de acumulación de sedimentos finos. Está ubicada a $1720 \mathrm{~m}$ de altitud y presenta un bosque con árboles de gran porte como: Coccoloba sp. (Polygonaceae), Cordia panamensis (Boraginaceae), Coussapoa duquei (Urticaceae), Dendropanax cf. macrophyllus (Araliaceae) y Ficus sp. (Moraceae), con un dosel cercano a los $35 \mathrm{~m}$. En esta estación de muestreo se registraron 527 individuos en total en un área de $2500 \mathrm{~m}^{2}, 57$ corresponden a árboles con una altura mayor a
$1.3 \mathrm{~m}$ y DAP mayor a $5 \mathrm{~cm}$, donde las especies más abundantes corresponden a las familias Araceae (Anthurium sp.), Piperaceae (Piper sp.), Flacourtiaceae y palmas de la familia Arecaceae (Chamaedorea pinnatifrons). Los hábitos de crecimiento hierbas terrestres y arbustos fueron predominantes en esta estación (Bedoya \& Orozco, 2015).

Quebrada Don Alonso: Estación 5 (E5, ver Anexo 1), ubicada a una altitud de $1849 \mathrm{~m}$ $\left(05^{\circ} 01^{\prime} 5079\right.$ ' N - 75³1'39.59” W) (Fig. 1), con un ancho de $\sim 1.26 \mathrm{~m}$, una profundidad de $\sim 7.03 \mathrm{~cm}$ y un caudal de $\sim 0.02 \mathrm{~m}^{3} / \mathrm{s}$, predomina el sustrato roca y sedimento fino, la hojarasca está casi ausente y la que se encuentra es de la especie Bambusa sp. (Poaceae). Corresponde a la primera quebrada con impacto agrícola, donde existe extracción de guadua y presencia de cultivos de hortalizas en el predio inmediato a la quebrada. Hay presencia de algunos árboles de las familias Cecropiaceae (Cecropia angustifolia) con una altura máxima de $30 \mathrm{~m}$ y Asteraceae (Montanoa quadranglaris). Los hábitos de crecimiento predominantes fueron hierbas y arbustos de las familias Piperaceae (Piper cf. crassinervium), Urticaceae (Urera baccifera), Poaceae y Solanaceae; en total se registraron 206 individuos en un área de $2500 \mathrm{~m}^{2}$, donde solo siete corresponden a árboles con una altura mayor a $1.3 \mathrm{~m}$ y DAP mayor a $5 \mathrm{~cm}$ (Bedoya \& Orozco, 2015).

Quebrada Don Fernando: Estación 6 (E6, ver Anexo 1), corresponde a la segunda quebrada impactada por agricultura $\left(05^{\circ} 01^{\prime} 36.9^{\prime \prime} \mathrm{N}\right.$ $75^{\circ} 31$ '26.3” W) a una altitud de $1754 \mathrm{~m}$ (Fig. $1)$, con un ancho de $\sim 1.01 \mathrm{~m}$ y una profundidad de $\sim 6.42 \mathrm{~cm}$, presenta un caudal de $\sim 0.04 \mathrm{~m}^{3} / \mathrm{s}$, predomina el sustrato sedimento fino, seguido de roca de tamaño medio $(15 \sim 20 \mathrm{~cm})$, la hojarasca está casi ausente. Los hábitos de crecimiento predominantes fueron hierbas terrestres y arbustos, con especies de las familias Melastomataceae (L. subseriata), Asteraceae ( $M$. quadranglaris), Zingiberaceae (Hedychium coronarium) y Poaceae (Pennisetum sp.). En total se registraron 151 individuos, de cuales 14 
corresponden a árboles con una altura mayor a $1.3 \mathrm{~m}$ y DAP mayor a $5 \mathrm{~cm}$; donde se destaca la presencia de las familias Lauraceae (Nectandra cf. reticulata) y Urticaceae junto al cultivo de hortalizas con una altura promedio de $15 \mathrm{~m}$ (Bedoya \& Orozco, 2015). En esta estación la expansión de la frontera agrícola llega justo hasta el cauce de la quebrada, lo cual eliminó la vegetación ribereña.

Diseño de muestreo: Se realizaron seis muestreos en cada una las estaciones elegidas, abarcando los meses de altas lluvias (febrero y noviembre 2014 y febrero 2015), lluvias intermedias (julio 2014) y bajas lluvias (abril y septiembre 2014). La recolecta de los insectos acuáticos se realizó mediante el uso de una red Surber $(30.5 \times 30.5 \mathrm{~cm})$ con ojo de malla de $250 \mu \mathrm{m}$, muestreando los sustratos más representativos al interior del cauce (sedimento, roca y hojarasca), con tres repeticiones por cada tipo de sustrato. Los insectos recolectados se almacenaron y fijaron en alcohol al $96 \%$ para su posterior identificación hasta el menor nivel taxonómico posible, utilizando las claves de Roldán (1996), Domínguez, Molineri, Pescador, Hubbard y Nieto (2006), Bonada, Rieradevall y Prat (2007), Merritt et al. (2008) y Domínguez y Fernández (2009). La identificación del material recolectado se llevó a cabo en el laboratorio de Colecciones Biológicas de la Universidad de Caldas y los individuos fueron depositados en la Colección Entomológica del programa de Biología de la Universidad de Caldas - CEBUC (Registro Humboldt: No 178).

Variables físicas, químicas e hidrológicas: En tres de los seis muestreos (febrero, julio y noviembre 2014) se midieron in situ las variables físicas: temperatura del agua $\left({ }^{\circ} \mathrm{C}\right), \mathrm{pH}$, conductividad $(\mu \mathrm{S})$ y oxígeno disuelto $(\mathrm{mg} / \mathrm{L})$. Se tomaron muestras de agua de cada quebrada en tres de los seis muestreos (febrero, julio y noviembre 2014), para la posterior determinación en el laboratorio de las variables químicas: demanda química de oxígeno (DQO), demanda biológica de oxígeno
$\left(\mathrm{DBO}_{5}\right)$, coliformes totales, coliformes fecales, sólidos totales (ST), sólidos suspendidos totales (SST), cianuro $(\mathrm{CN})$, boro $(\mathrm{B})$, plomo $(\mathrm{Pb})$, mercurio $(\mathrm{Hg})$, nitrógeno amoniacal $\left(\mathrm{NH}_{3}-\mathrm{N}\right)$, fosfatos $\left(\mathrm{PO}_{4}\right)$, sulfatos $\left(\mathrm{SO}_{4}\right)$, hierro $(\mathrm{Fe})$, cloruros $(\mathrm{Cl}-)$, grasas y aceites, nitratos $\left(\mathrm{NO}_{3}\right)$, nitritos $\left(\mathrm{NO}_{2}\right)$ y aluminio (Al) (Anexo 1), todas las muestras fueron tomadas siguiendo los protocolos para la toma y preservación de muestras fisicoquímicas de agua (debidamente refrigeradas), el análisis de las variables fisicoquímicas fue realizado por el laboratorio de calidad de agua ACUATEST S.A. Las variables hidrológicas profundidad $(\mathrm{cm})$, ancho $(\mathrm{m}) \mathrm{y}$ velocidad del cauce $(\mathrm{m} / \mathrm{s})$ se tomaron in situ durante los seis meses de muestreo, según lo propuesto por Chará (2003), para la evaluación biológica de ambientes acuáticos.

La comunidad de insectos acuáticos se evaluó considerando tres variables de respuesta (riqueza, abundancia y composición). Para la evaluación general de la comunidad de insectos acuáticos, se determinó la completitud de muestreo ( $\hat{C} n)$ en cada quebrada, siguiendo la metodología propuesta por Chao y Jost (2012). La Ĉn indica la proporción "total" de la comunidad representada por las morfoespecies capturadas (Chao \& Jost 2012)

$$
\hat{\mathrm{C}} \mathrm{n}=\frac{1+f 1}{n} *\left[\frac{((n-1) f 1)}{((n-1) f 1+2 f 2)}\right],
$$

donde $n$ es la abundancia total de la muestra, f1 y f2 corresponden a las morfoespecies con uno (singletons) y dos (doubletons) individuos respectivamente. Cuando $\hat{\mathrm{C}} \mathrm{n} \approx 100 \%$ el muestreo está completo con respecto al esfuerzo y técnica de captura usada (Chao \& Jost, 2012). La diversidad de los insectos acuáticos se comparó por medio de la diversidad de orden $\mathrm{q}\left({ }^{\mathrm{G} D}\right)$ (Chao et al., 2014), la cual se basa en el número efectivo de especies

$$
{ }^{q} D \equiv\left(\sum_{i=1}^{S} p_{i}^{q}\right)^{1 /(1-q)} .
$$

Donde el valor de q determina la influencia de la abundancia de las morfoespecies sobre los valores de diversidad (Chao et al., 2014). En este trabajo se usaron tres valores, $\mathrm{q}=0$ 
(riqueza de especies), $\mathrm{q}=1$ (exponencial del índice de Shannon) y q $=2$ (recíproco del índice de Simpson) (Moreno, Barragán, Pineda, \& Pavón, 2011). La estimación y comparación de qD se basa en la comparación de la diversidad bajo el mismo nivel de cobertura de muestreo entre comunidades (Chao et al., 2014). Las diferencias significativas se obtienen considerando el traslape de los intervalos de confianza (I.C) $95 \%$ (Chao et al., 2014; Cumming, Fidler, \& Vaux, 2007). Estos análisis se realizaron con el paquete "iNEXT" del programa estadístico R 3.3.0 (R Core Team, 2015).

Con el fin de evaluar el efecto del tipo de impacto y tipo de sustrato sobre la riqueza (S) y abundancia (N) de insectos acuáticos, se utilizaron modelos lineales generalizados mixtos (GLMM, por sus siglas en ingles). Estos modelos combinan propiedades de métodos estadísticos usados ampliamente en ecología, como los modelos lineales mixtos (MLM) y los modelos lineales generalizados (MLG) (Bolker et al., 2008), los cuales constituyen una excelente herramienta para el análisis de datos no normales, donde se combinan factores con efectos fijos (tipo de impacto y sustrato) y aleatorios (réplica anidada a la quebrada) (Bolker et al., 2008; Bates, 2010; Seoane, 2014). Tras realizar la exploración de los datos se empleó un único modelo lineal generalizado mixto (GLMM), con la función "glmer" del paquete "Ime4" de R v.3.3.0 (R Core Team 2015): glmer $(Y \sim$ Impacto + Sustrato + (Replica|Quebrada, data = abundancia, family = "poisson"), donde $Y$ es la variable respuesta (riqueza o abundancia); como factor aleatorio se estableció la quebrada (anidada a la réplica) y como efectos fijos el tipo de impacto y tipo de sustrato. Posteriormente, a la aplicación de los modelos se probó la normalidad de los residuales de forma gráfica para verificar su ajuste.

Para determinar la relación que existe entre las variables fisicoquímicas, hidrológicas y la abundancia de insectos acuáticos, se realizó un análisis de correspondencia canónica (ACC) con el software Canoco 4.5 (ter Braak \& Šmilauer, 2002). Este análisis se realizó con las variables hidrológicas $\left(\mathrm{O}_{2}\right.$, caudal, $\mathrm{pH}$ y
Conductividad) y fisicoquímicas (DQO, ST, $\mathrm{NO}_{2}, \mathrm{SO}_{4}, \mathrm{Fe}, \mathrm{Cl}$ - y $\mathrm{PO}_{4}$ ), estas fueron elegidas de acuerdo a su significancia por medio de la prueba de Monte Carlo ( $\mathrm{p} \leq 0.05$ ), adicionalmente, se tuvo en cuenta la altitud de cada una de las quebradas (estaciones de muestreo). El análisis se realizó con datos de tres de los seis muestreos, ya que las variables fisicoquímicas fueron medidas únicamente en tres eventos de muestreo. La evaluación de la composición se realizó por medio de un análisis de correspondencia simple (ACS), con el fin de determinar si la presencia de las morfoespecies de insectos acuáticos, depende de un tipo de impacto en particular. Este análisis permite realizar comparaciones gráficas e identificar asociaciones entre dos variables cualitativas (Greenacre, 1984); esta prueba estadística fue realizada con el programa STATISTICA 8.0 (StatSoft, 2007).

Las diferencias en la composición de la comunidad de insectos acuáticos respecto a los factores tipo de impacto, época climática y sustrato, se analizaron mediante un análisis de varianza multivariado, con base en permutaciones (PERMANOVA), usando el índice de Bray-Curtis y 999 permutaciones (Anderson, 2001; McArdle \& Anderson, 2001), este análisis multivariante tiene en cuenta la abundancia de cada taxón y no solo la abundancia total. En caso de encontrar diferencias significativas, se identificaron los taxa que más contribuyeron a estas diferencias mediante un análisis de porcentaje de similitud (SIMPER) (Clarke, 1993). Estos análisis se realizaron con la función "adonis" del paquete "vegan" en el programa estadístico R 3.3.0 (R Core Team, 2015), usando 100 aleatorizaciones, bajo un $\alpha=0.05$.

\section{RESULTADOS}

Se recolectaron 12502 individuos agrupados en nueve órdenes, 41 familias y 85 morfoespecies (Anexo 2), donde los géneros Simulium, Baetodes, Cricotopus y Andesiops fueron los más abundantes (64.2\%), seguidos de un segundo grupo conformado por los géneros Smicridea, Leptohyphes, Camelobaetidius, Nanomis, Atopsyche, Anacroneuria, 
Anchytarsus y Helicopsyche, y las morfoespecies Chironominae sp.1, Tanytarsini sp.1 y Tanypodinae sp.1 (Chironomidae), los cuales representan el $21.2 \%$. En general y con respecto a los impactos evaluados, el $44 \%$ de la abundancia se registró en las zonas de referencia, $28.8 \%$ en las zonas con impacto minero y el $27.4 \%$ en las zonas con impacto agrícola (Anexo 2). La abundancia en las estaciones con impacto agrícola estuvo dominada por individuos de las familias Simuliidae (Simulium) y Chironomidae (Cricotopus, Chironominae sp.1), mientras que en las estaciones con impacto minero hubo mayor riqueza de morfoespecies (Fig. 2) y la abundancia presentó valores más altos (Anexo 2). Con respecto a los sustratos, la hojarasca sustentó el $45 \%$ de la abundancia total, seguido por el sustrato roca $(42.2 \%)$ y por último el sedimento fino con $11.2 \%$; la abundancia estuvo dominada en los tres sustratos por Baetodes, Cricotopus y Simulium; en términos de riqueza el patrón se repitió, y en la hojarasca estuvieron representadas por un mayor número de morfoespecies (86.3\%), seguido por el de roca $(73.7 \%)$ y de sedimento fino $(53.7 \%)$.

\section{Representatividad de muestreo y diver-} sidad ( ${ }^{\mathrm{q} D}$ ): En todos los casos se alcanzó $\sim 100 \%$ de completitud, lo que indica que el esfuerzo de muestreo realizado en este trabajo fue suficiente para registrar la mayoría de las morfoespecies presentes en estos ecosistemas acuáticos. Se asumió una completitud del $100 \%$ para todas las estaciones y se compararon de forma directa las medidas de diversidad ( ${ }^{\mathrm{q} D}$ ) entre ellas. En ambas localidades (Gallinazo y Llanitos) la riqueza de especies $\left({ }^{0} \mathrm{D}\right)$ presentó una tendencia decreciente desde las zonas de referencia hacia las estaciones

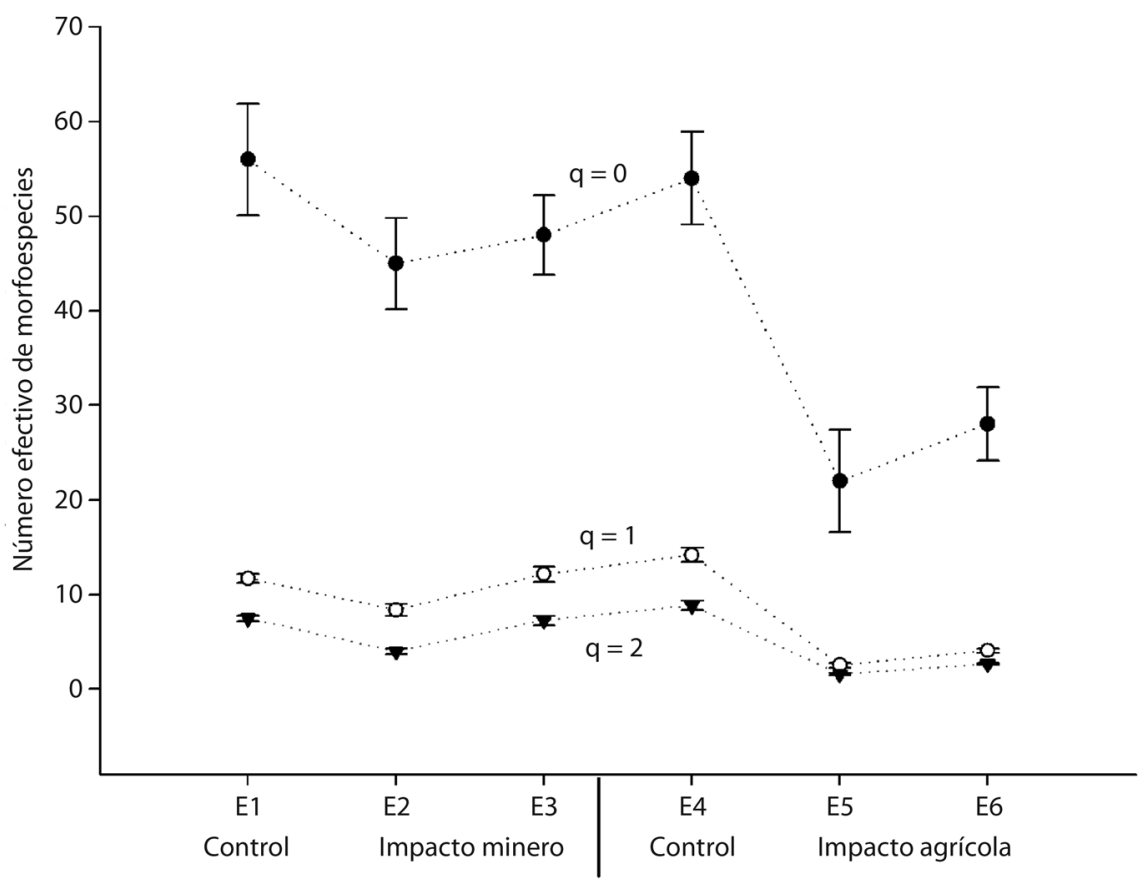

Fig. 2. Perfiles de diversidad para cada una de las estaciones evaluadas. Donde $q=0$ : riqueza de morfoespecies, $q=1$ : diversidad per se y q = 2: toma en cuenta únicamente las morfoespecies más abundantes (I.C = 95\%). Se presentan las zonas control y las zonas impactadas por minería y agricultura.

Fig. 2. Diversity profiles for each of the evaluated stations. Where $q=0$ : species richness, $q=1$ : diversity per se and $q=2$ : takes into account only the most abundant morphospecies $(C . I=95 \%)$. The control and altered zones by mining and agriculture are shown. 
impactadas por minería y/o agricultura (Fig. 2), dicha tendencia no fue significativa para la localidad Gallinazo (impactada por minería), pero si lo fue para la localidad Llanitos (impactada por agricultura), donde la zona de referencia presentó una mayor riqueza con respecto a las dos zonas con impacto agrícola (relación $\sim 2: 1$ ). En el caso de la ${ }^{1} \mathrm{D}$ y ${ }^{2} \mathrm{D}$, no se observó una tendencia de respuesta y la diversidad presentó un patrón más o menos homogéneo entre estaciones de muestreo. En la localidad Llanitos, la estación E4 presentó diferencias significativas en términos de la ${ }^{1} \mathrm{D}$ $\mathrm{y}{ }^{2} \mathrm{D}$ con respecto a las estaciones impactadas por agricultura (E5 y E6), donde E4 es casi tres veces ( 3:1) más diversa que E5 y E6 (Fig. 2).

Impacto agrícola y minero sobre la comunidad de insectos acuáticos: El tipo de impacto afectó la riqueza de morfoespecies (GMLM, $\mathrm{z}=-3.481, \mathrm{p}=0.0005)$ y las quebradas con impacto agrícola presentaron una menor riqueza de insectos; adicionalmente, el sustrato no alteró la riqueza de insectos (GMLM, $\mathrm{z}=-1.744, \mathrm{p}=0.0812$ ), pero el sustrato hojarasca siempre presentó el mayor número de morfoespecies. Al evaluar la abundancia, el modelo demuestra la importancia del tipo de impacto (GMLM, z $=-3.333, \mathrm{p}=$ 0.0008 ) para la comunidad de insectos acuáticos, donde la zona impactada por agricultura presentó una reducción en el número de individuos. Para el factor sustrato también se presentaron diferencias significativas (GMLM, $\mathrm{z}=5.005, \mathrm{p}=0.0000$ ). En términos generales, el impacto agrícola presentó los valores más bajos de riqueza y abundancia, este mismo patrón se repitió al evaluar los sustratos, donde el sedimento fino fue el que presentó el menor número de individuos y morfoespecies, $\mathrm{y}$ fue el sustrato predominante al interior del cauce en las estaciones impactadas por agricultura.

El análisis de correspondencia canónica (ACC) indica que las variables temperatura $\left(\mathrm{T}^{\circ}\right)$, altitud (Alt), caudal $(\mathrm{Q})$, conductividad (Con), hierro $(\mathrm{Fe})$ oxígeno disuelto $\left(\mathrm{O}_{2}\right)$ y $\mathrm{pH}$ determinaron la conformación de la comunidad de insectos acuáticos en las estaciones evaluadas (Fig. 3) y en menor medida las variables sulfatos $\left(\mathrm{SO}_{4}\right)$, fosfatos $\left(\mathrm{PO}_{4}\right)$, nitritos $\left(\mathrm{NO}_{2}\right)$, cloruros $(\mathrm{Cl})$, demanda química de oxígeno (DQO) y sólidos totales (ST). La estructura de la comunidad de insectos de las zonas con impacto minero (estaciones E1, E2 y E3) estuvo relacionada positivamente con las variables: caudal, altitud y oxígeno disuelto, las cuales presentaron los valores más altos, y de manera negativa con las variables $\mathrm{pH}$ y temperatura (T) (Anexo 1). Las variables fosfatos, cloruros, nitratos, sulfatos y sólidos totales también pueden determinar la estructura de la comunidad y a su vez tener una estrecha relación con la conductividad (Fig. 3). La estación E2 (impactada por minería) en los muestreos uno y tres, estuvo relacionada positivamente con la conductividad.

En cuanto a la zona impactada por agricultura, no se presentó un patrón de agrupamiento consistente entre estaciones, la composición de insectos acuáticos estuvo relacionada de forma positiva con las variables: temperatura, $\mathrm{pH}$ y hierro, y negativa con la conductividad. Las estaciones E6M1 y E6M3 tuvieron una relación negativa con las variables caudal, altitud y oxígeno disuelto por ser quebradas más pequeñas y encontrarse en zonas más bajas (Anexo 1, Fig. 3), en las demás estaciones esta relación no fue tan clara. La estación de referencia E4 estuvo relacionada positivamente con la temperatura y el $\mathrm{pH}\left(\sim 19^{\circ} \mathrm{C}\right.$ y 8.3 , respectivamente) y negativamente con las variables caudal, oxígeno disuelto y altitud $\left(0.09 \mathrm{~m}^{3} / \mathrm{s}, 2.75 \mathrm{mg} / \mathrm{L} \mathrm{y}\right.$ 1720 m, respectivamente) (Fig. 3). Las estaciones impactadas por agricultura (E5 y E6) estuvieron influenciadas por la temperatura y el $\mathrm{pH}$ (Fig. 3), aunque E5 y E6 en el muestreo dos se evidenció mayor influencia por el hierro, esta relación no fue clara. En términos generales, la composición de especies en las estaciones impactadas por agricultura presentó una estructura más sencilla en comparación con la zona minera, donde pocos géneros como Chimarra y Rhagovelia son abundantes, con dominancia de las familias Simuliidae y Chironomidae características de zonas alteradas (Zúñiga \& Cardona, 2009) (Anexo 2, Fig. 3). 


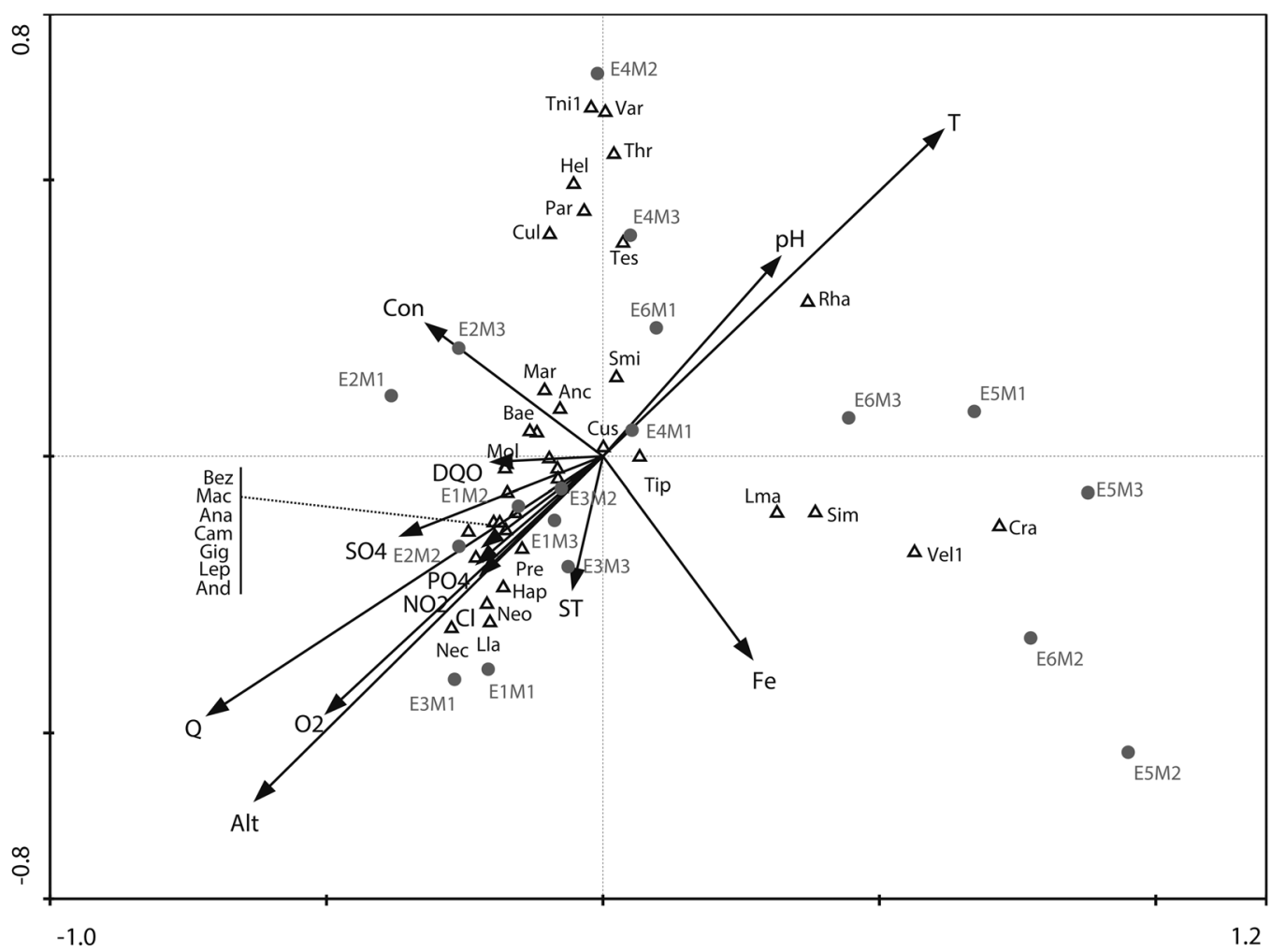

Fig. 3. Análisis de correspondencia canónica $(\mathrm{ACC}$, varianza explicada $=67.1 \%)$. Se muestra la asociación entre las morfoespecies de insectos acuáticos y las variables físico-químicas evaluadas en cada estación (ver códigos de las variables y morfoespecies en el Anexo 1 y Anexo 2, respectivamente). Los enunciados en color gris corresponden a: E = estación y $\mathrm{M}=$ muestreo.

Fig. 3. Canonical correspondence analysis $(\mathrm{CCA}$, explained variance $=67.1 \%)$. The association between morphospecies of aquatic insects and physico-chemical variables evaluated at each station is shown (see variable and morphospecies codes in Appendix 1 and Appendix 2, respectively). The labels in gray correspond to: $\mathrm{E}=$ station and $\mathrm{M}=$ sampling.

Análisis de composición de insectos acuáticos: Al evaluar la composición de los insectos acuáticos por medio del análisis de correspondencia simple (ACS), se presentaron tres agrupamientos entre las morfoespecies y las estaciones de muestreo, el análisis mostró que el $71.7 \%$ de la variación en la composición de morfoespecies estuvo explicada por la combinación de dos dimensiones. Las morfoespecies no se agrupan de forma equitativa entre las estaciones de muestreo y se forman tres grupos en función de la localidad. El primer grupo estuvo compuesto por las dos estaciones con influencia de actividad minera y su respectiva zona de referencia, donde los géneros más abundantes fueron Baetodes, Cricotopus y Andesiops (Fig. 4). La zona impactada por agricultura, se dividió en dos grupos, uno conformado por la estación E4 (referencia), donde los géneros Baetodes, Smicridea y Cricotopus fueron los más abundantes, también se observó que los géneros Thraulodes, Tricorythodes y Varipes (Ephemeroptera) fueron exclusivos de esta estación (Fig. 4), ya que solo se registraron en esta quebrada (E4) durante todos los muestreos. El segundo grupo estuvo constituido por las estaciones E5 y E6 con presencia de impacto agrícola, este resultado confirma nuevamente que estas estaciones con impacto agrícola eran las menos diversas (Fig. 4). Cabe destacar que la estación E4 sustenta una gran diversidad y está más asociada a las estaciones 


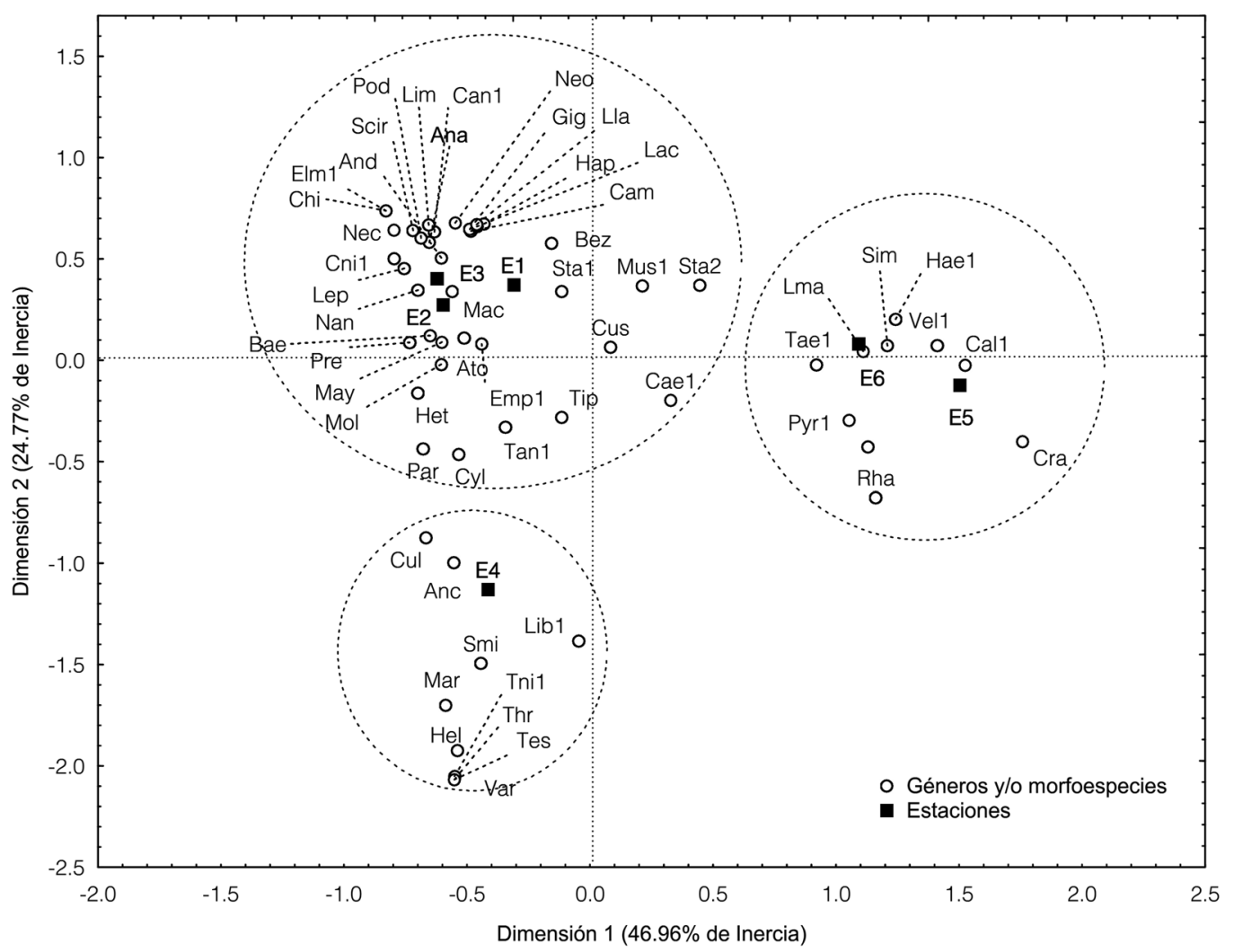

Fig. 4. Análisis de correspondencia simple (ACS) para las estaciones de muestreo evaluadas (Dimensión 1: $\mathrm{X}^{2}=7032.86$, $\mathrm{p}<0.000$; Dimensión 2: $\left.\mathrm{X}^{2}=3710.12, \mathrm{p}<0.000\right)$. Ver códigos de las morfoespecies en el Anexo 2. E1: control minería, E2 y E3: minería, E4: control agricultura, E5 y E6: agricultura.

Fig. 4. Simple correspondence analysis (SCA) for evaluated sampling stations (Dimension 1: $X^{2}=7032.86, p<0.000$; Dimension 2: $\left.\mathrm{X}^{2}=3710.12, \mathrm{p}<0.000\right)$. See morphospecies codes in Appendix 2. E1: mining control, E2 and, E3: mining, E4: agriculture control, E5 and, E6: agriculture.

de la localidad Gallinazo (impactada por minería) (Fig. 4).

La composición de la comunidad de insectos acuáticos se ve afectada de forma significativa por el tipo de impacto (PERMANOVA, $\mathrm{F}_{2,309}=21.82, \mathrm{p}=0.001$ ), sustrato (PERMANOVA, $\left.\mathrm{F}_{2,309}=2.84, \mathrm{p}=0.001\right)$ y muestreo (PERMANOVA, $\mathrm{F}_{1,309}=6.65, \mathrm{p}=0.001$ ). Por medio del SIMPER se identificaron los taxa que más contribuyeron a estas diferencias para cada uno de los factores. Para el factor tipo de impacto se encontró que los géneros Baetodes, Cricotopus, Andesiops, Smicridea, Simulium y Camelobaetidius contribuyeron entre un 62 70\% (Anexo 3); las contribuciones acumulativas estuvieron dominadas por Baetodes con 13.16 ind./prom en minería y 10.52 ind./ prom en el control (Cuadro 1). Se evidenció que Baetodes, Andesiops y Cricotopus son los géneros que más aportaron a las zonas control y con impacto minero, mientras en agricultura fue Simulium (Diptera: Simuliidae) típicos de zonas perturbadas.

En términos de sustrato, se encontró que los géneros Baetodes, Simulium, Cricotopus y Andesiops contribuyeron entre un 52 58\% a las diferencias encontradas en la composición entre los sustratos, donde Baetodes fue dominante en roca (15.55 ind./prom), Cricotopus en sedimento (3.69 ind./prom) y Simulium y Cricotopus en hojarasca (13.96 y 10.58 ind./prom, respectivamente). La contribución acumulativa 
CUADRO 1

Morfoespecies dominantes entre tipos de impacto

TABLE 1

Dominant morphospecies among types of impact

\begin{tabular}{lccc}
\multicolumn{1}{c}{ Morfoespecie } & Números de individuos promedio (ind/prom) & Agricultura \\
Baetodes & 10.52 & Minería & 0.02 \\
Andesiops & 4.06 & 13.16 & 0.01 \\
Cricotopus & 9.93 & 3.70 & 6.68 \\
Leptohyphes & 0.72 & 3.63 & 0.00 \\
Nanomis & 1.07 & 3.21 & 0.00 \\
Simulium & 3.44 & 2.01 & 21.30 \\
Chironominae sp.1 & 1.58 & 1.33 & 2.07 \\
Smicridea & 4.06 & 3.21 & 0.25 \\
Camelobaetidius & 3.04 & 0.5 & 0.00 \\
Atopsyche & 1.88 & 0.71 & 0.00 \\
Total & 40.3 & 0.44 & 30.33 \\
\hline
\end{tabular}

Las diferencias fueron halladas por medio del análisis SIMPER. Número de individuos promedio (ind/prom) por taxa. Differences were found by SIMPER analysis. The average number of individuals by taxa (ind/prom).

está determinada por los mismos cuatro géneros (Anexo 3). Al evaluar el factor muestreo (época climática) las mayores contribuciones acumulativas estuvieron dadas por los géneros Cricotopus, Simulium, Baetodes, Andesiops y Smicridea con una contribución entre 56 59\% para la época de altas lluvias, las contribuciones para la época de lluvias intermedias estuvieron dadas por Baetodes, Simulium, Cricotopus y Andesiops con una contribución del $\sim 61 \%$ y para la época de bajas lluvias los géneros Baetodes, Simulium, Cricotopus, Nanomis, Andesiops y Rhagovelia contribuyeron con cerca del $65 \%$; en términos generales, la mayor contribución estuvo dada generalmente por los mismos géneros (Anexo 3).

\section{DISCUSIÓN}

Las alteraciones de origen antrópico como la minería y la agricultura alteran directa e indirectamente la diversidad de insectos al interior de los ecosistemas acuáticos (Hepp et al., 2010; Bernhardt \& Palmer, 2011; Chará-Serna et al., 2015). En este estudio, la diversidad y composición de la comunidad de insectos acuáticos se ve afectada por la perturbación de tipo agrícola y minera, y el sustrato. En términos de diversidad ( $\left.{ }^{\mathrm{Q} D} \mathrm{D}\right)$, las zonas de referencia son las que presentan los valores más altos y las quebradas impactadas por minería sustentan una diversidad de insectos acuáticos muy similar a las quebradas de referencia, lo cual se debe a las características físicas e hidrológicas de las quebradas y a la presencia de la vegetación ribereña. Las zonas impactadas por minería presentaron una mayor velocidad del cauce y valores altos de $\mathrm{O}_{2}$, con respecto a las zonas impactadas por agricultura, lo cual hace que sean más similares a las zonas de referencia. Por otro lado, las estaciones con impacto minero cuentan con una franja amplia ( $\sim 20 \mathrm{~m})$ de vegetación ribereña, lo cual es un factor preponderante para el mantenimiento de las comunidades acuáticas (Hepp et al., 2010; Chará-Serna et al., 2015). Estos resultados concuerdan con lo reportado por varios autores, donde se reiteran los efectos adversos de la agricultura sobre los ecosistemas acuáticos y la diversidad (Maloney \& Weller, 2011; Piggott et al., 2012; Chará-Serna et al., 2015), y de la importancia de la vegetación ribereña 
para el mantenimiento de una mayor diversidad al interior de los ecosistemas acuáticos (Subramanian, Sivaramakrishnan, \& Gadgil, 2005).

La diversidad de insectos acuáticos puede estar determinada por la disponibilidad de recursos al interior del cauce, las características fisicoquímicas e hidrológicas (Ramírez \& Viña, 1998) y el tiempo de exposición al impacto (Wood \& Armitage, 1997). La presencia de vegetación ribereña provee mejores condiciones al interior de las quebradas, proporcionando mayor disponibilidad de recurso (refugio y alimento) para los insectos acuáticos (Subramanian et al., 2005), ya que ofrece alimento para muchos organismos y las raíces protegen al suelo de la erosión, y mantienen la integridad del cauce (Naiman, Décamps, \& McClain, 2005), lo cual explicaría que las quebradas impactadas por minería (con presencia de vegetación ribereña) sustentan una mayor diversidad y composición de insectos acuáticos, similar a las quebradas de referencia, con respecto a las de la zona agrícola. $\mathrm{La}$ mayor riqueza $(\mathrm{S})$ y abundancia $(\mathrm{N})$ vegetal se presentó en las zonas de referencia (El Establo: $\mathrm{N}=527, \mathrm{~S}=106$; Romerales: $\mathrm{N}=328$, $\mathrm{S}=130$ ), y en las impactadas por minería (California: $\mathrm{N}=292, \mathrm{~S}=88$; Toldafría: $\mathrm{N}=292$, $\mathrm{S}=70$ ) (Bedoya \& Orozco, 2015).

De este modo, la pérdida de la vegetación ribereña representa un factor determinante para la comunidad de insectos acuáticos, donde la deforestación ribereña en las estaciones impactadas por agricultura, abre el dosel, disminuye la sombra e incrementa la sedimentación en las quebradas, y a su vez se impide la autodepuración (Vannote, Minshall, Cummins, Sedell, \& Cushing, 1980; Hershey \& Lamberti, 1998). Este resultado concuerda con lo encontrado por Subramanian et al. (2005), quienes demuestran que en ecosistemas acuáticos con impacto agrícola, la distribución y la abundancia de los insectos acuáticos están principalmente influenciadas por la presencia o no de la vegetación ribereña.

En las quebradas con impacto minero, características como mayor caudal, concentración de oxígeno y altitud en estas quebradas con respecto a las quebradas con impacto agrícola, pueden determinar la comunidad de insectos (Giraldo, Chará, Zúñiga, Chará-Serna, \& Pedraza, 2014). La zona impactada por agricultura presentó temperaturas mucho mayores (18 $\sim 20^{\circ} \mathrm{C}$ ) en relación con las zonas de referencia y la zona minera $\left(13 \sim 19^{\circ} \mathrm{C}\right)$, lo cual tiene una relación directa con la altitud de cada localidad; el aumento de la temperatura y la mayor cantidad de sedimentos presente en estas quebradas constituye una clara perturbación para la comunidad de insectos acuáticos (Piggott et al., 2012). La velocidad del cauce influye en la dispersión y la permanencia de los insectos en las zonas de estudio; este fenómeno de arrastre de los individuos por la velocidad del cauce, es conocido como deriva y se ha documentado que es un proceso que implica que los individuos presenten adaptaciones a condiciones particulares de los cauces, y es importante para su dispersión y permanencia en las quebradas (Humphries, 2002). Lo anterior explicaría que la composición de morfoespecies no cambia de forma significativa con la época climática y los valores bajos de abundancia en las épocas de altas lluvias, lo cual es consistente con otros trabajos realizados en Colombia (RodríguezBarrios, Ospina-Torres, Gutiérrez, \& Ovalle, 2007; Longo, Zamora, Guisande, \& Ramírez, 2010; Tamaris-Turizo, Rodríguez-Barrios, \& Ospina, 2013), donde el efecto de la deriva por el aumento del caudal, y la presencia o ausencia de la vegetación ribereña estarían modulando la comunidad de insectos acuáticos.

Adicionalmente, el efecto del impacto sobre las comunidades puede estar determinado por el tiempo de uso del suelo y de recuperación del impacto (Wood \& Armitage, 1997). En este estudio, la agricultura se ha llevado a cabo por $\sim 90$ años y la minería $\sim 50$ años (com. pers.); además, las zonas de referencia para cada localidad se han mantenido sin perturbación antrópica por el mismo periodo de tiempo ( $\sim 90 \mathrm{y} \sim 50$ años, respectivamente). Aunque la minería para la extracción de oro tiene efectos nocivos sobre las comunidades acuáticas por el uso de mercurio y cianuro (Defensoría del Pueblo Colombia, 2010), estos efectos no fueron muy evidentes 
en este estudio. Lo anterior puede ser debido a que las estaciones a las que se tuvo acceso (por cuestiones de seguridad) para muestreo, estaban ubicadas a una distancia de aproximadamente $2 \mathrm{~km}$ de la mina y considerando que los ríos son sistemas dinámicos, probablemente las sustancias toxicas derivadas de dicha actividad no tienen flujo continuo y se depositan rápidamente en el sedimento (Ferreira da Silva et al., 2009; Marrugo-Negrete \& Paternina-Uribe, 2011). Además, posiblemente la autodepuración de las quebradas en la localidad minera esté disminuyendo el efecto de los vertimientos en el cauce, lo cual hace que el tiempo de uso y el impacto por minería no estén afectando drásticamente la comunidad de insectos acuáticos. Por su parte, la agricultura, tiene efectos sobre la diversidad por el tipo de uso (los cultivos son inmediatos a la fuente de agua), la escorrentía de nutrientes (Piggott et al., 2012), el uso de pesticidas (Laabs et al., 2002; Clarke, Mac Nally, Bond, \& Lake, 2008; Maloney \& Weller, 2011; Chará-Serna et al., 2015) y el tiempo de uso ( 90 años; Wood \& Armitage, 1997), los cuales afectan de forma drástica a la comunidad de insectos acuáticos.

Los sustratos hojarasca y roca fueron abundantes en las estaciones de referencia y las impactadas por minería, mientras que en las estaciones impactadas por agricultura estos sustratos estaban casi ausentes y dominaba el sustrato sedimento, debido a la pérdida de la vegetación ribereña, estas condiciones favorecen la presencia de morfoespecies del orden Diptera (e.g. Cricotopus) típicos de zonas perturbadas (Zúñiga \& Cardona, 2009). Por otro lado, en la estación de referencia de la zona agrícola (E4), se encontraron tres géneros exclusivos de Ephemeroptera (Tricorythodes, Thraulodes y Varipes), los cuales están relacionados con ecosistemas acuáticos en buen estado de conservación (Domínguez \& Fernández, 2009; Zuñiga \& Cardona, 2009); Thraulodes y Tricorythodes son géneros bien representados en las quebradas andinas y tienen una amplia distribución en todo el departamento de Caldas (Gutiérrez \& G-Dias, 2015). En las estaciones agrícolas la composición es dominada por
Simulium, Cricotopus y Chironominae sp.1, todos tolerantes a la contaminación (Zúñiga \& Cardona, 2009).

La similitud en términos de composición de las zonas de referencia con las zonas mineras, revela la gran capacidad de estos ecosistemas de sustentar una gran diversidad biológica, donde se destaca la presencia y buena representatividad de morfoespecies de Ephemeroptera, Plecoptera y Trichoptera, relacionados con las buenas condiciones de los ecosistemas (e.g. mayor $\mathrm{O}_{2}$ disuelto, flujo permanente del agua) (Feminella, 1996; Smith, Wood, \& Gunn, 2003). En este estudio, se encontró que la composición de morfoespecies no cambia de forma significativa con la época climática, sin embargo, las pequeñas variaciones que se presentan, implican que las lluvias (precipitación mensual) pueden favorecer la permanencia de algunas morfoespecies con respecto a otras. Sin embargo, es posible que la velocidad del cauce sea mucho más determinante para los insectos acuáticos, ya que influye en la dispersión y la permanencia de los insectos en las zonas de estudio.

Los resultados de este trabajo concuerdan con la idea de que la diversidad de insectos acuáticos expuestos a actividades de tipo antrópico presentan una reducción con respecto a zonas conservadas, este patrón de respuesta ya ha sido reportado (Riseng, Wiley, Black, \& Munn, 2011; Giraldo et al., 2014; Edegbene, Arimoro, Odoh, \& Ogidiaka, 2015), donde la riqueza y la abundancia generalmente presentan sus valores más altos en sitios sin evidencia de perturbación. Además, Smith y Lamp (2008) argumentan que la estructura y composición de la comunidad de insectos acuáticos está más fuertemente influenciada por el tipo de impacto, que por otros factores como la época climática o el tipo de sustrato, aunque la diversidad de insectos acuáticos siempre tuvo una relación directa con la disponibilidad y tipo de sustrato, y en este estudio, el sustrato hojarasca fue el que sustentó la mayor riqueza y abundancia, seguido del sustrato roca; la presencia del sustrato hojarasca está directamente relacionado con los detritos que ingresan a las quebradas 
desde la vegetación ribereña, los cuales constituyen una importante fuente de recurso para los insectos acuáticos, y a su vez, juegan un papel importante en la determinación de la diversidad (Allan, 1995).

En conclusión, se encontró que las actividades antropogénicas (i.e. minería y agricultura) conllevan a cambios en la vegetación ribereña, variables como caudal y tipos de sustratos al interior del cauce, los cuales afectan la comunidad de insectos acuáticos en las quebradas andinas evaluadas, donde la agricultura es la actividad antrópica que representa un mayor impacto para la comunidad de insectos. La perturbación de tipo agrícola y minera alteran los ecosistemas de todo el mundo y no es posible generalizar el efecto de estas actividades sobre la comunidad de insectos acuáticos, ya que los usos del suelo y gran variedad de condiciones cambian entre localidades de estudio (Cooper, 1993; Laabs et al., 2002; Allan, 2004; Jergentz, Mugni, Bonetto, \& Schulz, 2004; Clarke et al., 2008; Maloney \& Weller, 2011; Torres \& Ramírez, 2014; Chará-Serna et al., 2015). Por lo tanto, los resultados de este trabajo ofrecen información acerca de la respuesta de la comunidad de insectos acuáticos ante la perturbación agrícola y minera en quebradas andinas de Caldas, Colombia, y se resalta la importancia de la vegetación ribereña en los ecosistemas acuáticos de los Andes colombianos, la cual permite que se presente una mayor biodiversidad. En términos generales, las alteraciones son factores importantes para la estructura de la comunidad en ecosistemas dulceacuícolas (Stanley \& Fisher, 1992), aunque la diversidad de insectos acuáticos puede estar modulada por factores como la presencia de vegetación ribereña, tipo y cantidad de sustratos al interior de la quebrada (al ser fuente de recurso) y el tipo de impacto.

\section{AGRADECIMIENTOS}

Al proyecto "Evaluación del impacto minero, agrícola y ganadero, mediante respuestas ecológicas y genéticas de macroinvertebrados acuáticos" (Código 0886913) financiado por Colciencias. Al equipo de trabajo del Laboratorio de Colecciones Biológicas de la Universidad de Caldas. A Aguas de Manizales S.A. E.S.P. por permitirnos trabajar en los predios de la quebrada Romerales. A Jonathan Giraldo por la elaboración del mapa de la zona de estudio y a todas las personas que contribuyeron en la ejecución de este trabajo. A los grupos de investigación: en Biodiversidad y Recursos Naturales (Bionat), Ecosistemas Tropicales y Genética, Biodiversidad y Manejo de Ecosistemas (Gebiome).

\section{RESUMEN}

Los ecosistemas dulceacuícolas están sometidos a fuertes presiones de tipo antrópico (e.g. agricultura y minería), las cuales ocasionan efectos adversos sobre la diversidad de insectos acuáticos. Se evaluó la comunidad de insectos en quebradas impactadas por agricultura y minería, y se determinó cómo estas actividades afectan a la comunidad. Se estudiaron seis quebradas en Caldas (Colombia), dos impactadas por minería, dos por agricultura y dos quebradas usadas como zonas de referencia. Los insectos fueron recolectados usando una red Surber con tres réplicas por tipo de sustrato (sedimento fino, roca y hojarasca). En total se recolectaron 12502 insectos acuáticos. Los géneros más representativos fueron: Baetodes, Simulium y Cricotopus (57.7 \%). Las quebradas impactadas por agricultura tienen baja diversidad de insectos acuáticos, comparado con las impactadas por minería $\left(\mathrm{F}_{2,309}=\right.$ $21.82, p=0.001)$. La zona agrícola presenta una estructura dominada por Simulium y Cricotopus (Diptera, morfoespecies tolerantes a la contaminación). Baetodes dominó en el sustrato roca, Cricotopus en el sedimento y Simulium y Cricotopus en la hojarasca $\left(\mathrm{F}_{2,309}=2.84, \mathrm{p}=0.001\right)$. Por lo tanto, ambos factores (tipo de impacto y sustrato) son importantes para determinar la diversidad de insectos acuáticos. Nuestros resultados sugieren que la actividad agrícola tiene un mayor impacto sobre la diversidad de insectos acuáticos, los cuales pueden estar asociados con la pérdida de la vegetación ribereña.

Palabras clave: ecosistemas acuáticos, Andes colombianos, alteración antrópica, insectos bentónicos, ecología, perturbación antrópica.

\section{REFERENCIAS}

Allan, J. D. (1995). Stream Ecology: Structure and function of running waters. Chennai: Chapman \& Hall.

Allan, J. D. (2004). Landscapes and riverscapes: the influence of land use on stream ecosystems. Annual 
Review of Ecology, Evolution, and Systematics, $35,257-284$.

Anderson, M. J. (2001). A new method for non-parametric multivariate analysis of variance. Austral Ecology, $26,32-46$.

Armenteras, D., \& Rodríguez, N. (2014). Dinámicas y causas de deforestación en bosques de Latino América: una revisión desde 1990. Colombia Forestal, 12(2), 233-246.

Bann, L., Alkemade, R., \& Koellner, T. (2013). Land use impacts on biodiversity in LCA: a global approach. The International Journal of Life Cycle Assessment, 18, 1216-1230.

Barbour, M. T., Gerritsen, J., Snyder, B. D., \& Stribling, J. B. (1999). Rapid Bioassessment Protocols for Use in streams and wadeable rivers: periphyton, Benthic Macroinvertebrates and Fish. Second Edition. Washington: US Environmental Protection Agency.

Bates, D. M. (2010). Lme4: Mixed-effects modeling with R. Wisconsin: Springer.

Bedoya, O. A., \& Orozco, J. A. (2015). Estructura y composición florística de parches de bosques asociados a ganadería, agricultura y minería en la cuenca del río Chinchiná (Tesis de Pregrado). Universidad de Caldas. Manizales, Colombia.

Bernhardt, E. S., \& Palmer, M. A. (2011). The environmental costs of mountaintop mining valley fill operations for aquatic ecosystems of the Central Appalachians. Annals of the New York Academy of Science, 1223, 39-57.

Bjerklie, D. M., \& LaPerriere, J. D. (1985). Gold-mining effects on stream hydrology and water quality, circle quadrangle, Alaska. Water Resources Bulletin, 21(2), 235-243.

Bolker, B. M., Brooks, M. E., Clark, C. J., Geange, S. W., Poulsen, J. R., Henry, M., Stevens, H., \& White, J. S .S. (2008). Generalized linear mixed models: a practical guide for ecology and evolution. Trends in Ecology and Evolution, 24(3), 127-135.

Bonada, N., Rieradevall, M., \& Prat, M. (2007). Macroinvertebrate community structure and biological traits related to flow permanence in a Mediterranean river network. Hydrobiologia, 589(1), 91-106.

Boudou, A., Maury-Brachet, R., Coquery, M., Durrieu, G., \& Cossa, D. (2005). Synergic effect of gold mining and damming on mercury concentration in fish. Environmental Science and Technology, 39, 2448-2454.

Brosse, S., Grenouillet, G., Gevrey, M., Khazraie, K., \& Tudesque, L. (2011). Small-scale gold mining erodes fish assemblage structure in small neotropical streams. Biodiversity and Conservation, 20, 1013-1026.
Canton, S. P. \& Ward, J. V. (1981). The aquatic insects, with emphasis on Trichoptera, of a Colorado stream affected by coal strip-mine drainage. The Southwestern Naturalist, 25(4), 453-460.

Chao, A. \& Jost, L. (2012). Coverage-based rarefaction and extrapolation: standardizing samples by completeness rather than size. Ecology, 93(12), 2533-2547.

Chao, A., Gotelli, N. J., Sieh, C. H., Sander, E. L., Ma. K. W., Colwell, R. K., \& Ellison, A. M. (2014). Rarefaction and extrapolation with Hill numbers: a framework for sampling and estimation in species diversity Studies. Ecological Monographs, 84(1), 45-67.

Chará, J. (2003). Manual para la evaluación biológica de ambientes acuáticos en microcuencas ganaderas. Cali: Fundación Centro para la Investigación en Sistemas Sostenibles de Producción Agropecuaria (CIPAV).

Chará-Serna, A. M., Chará, J., Zúñiga, M. C., Pedraza, G. X., \& Giraldo, L. P. (2010). Clasificación trófica de insectos acuáticos en ocho quebradas protegidas de la ecorregión cafetera colombiana. Universitas Scientiarum, 15(1), 27-36.

Chará-Serna, A. M., Chará, J., Giraldo, L. P., Zúñiga, M. C., \& Allan, J. D. (2015). Understanding the impacts of agriculture on Andean stream ecosystems of Colombia: a causal analysis using aquatic macroinvertebrates as indicators of biological integrity. Freshwater Science, 34, 727-740.

Clarke, K. R. (1993). Non-parametric multivariate analyses of changes in community structure. Australian Journal of Ecology, 18, 117-143.

Clarke A., Mac Nally, R., Bond, N., \& Lake, P. S. (2008). Macroinvertebrate diversity in headwater streams: a review. Freshwater Biology, 53, 1707-1721.

Cooper, C. M. (1993). Biological effects of agriculturally derived surface water pollutants on aquatic systems-a review. Journal of Environmental Quality, 22, 402-408.

Coutinho, H., Noellemeyyer, E., Jobbagy, E., Jonathan, M., \& Paruelo, J. (2009). Impacts of land use change on ecosystems and society in the Rio de la Plata basin. Applying Ecological Knowledge to Landuse Decisions. InterAmerican Agency for Global Change Research, 56-65.

Cumming, G., Fidler, F., \& Vaux, D. L. (2007). Error bars in experimental biology. The Journal of Cell Biology, 21, 7-11.

Defensoría del Pueblo Colombia. (2010). Minería de hecho en Colombia. Bogotá: Defensoría Delegada para los Derechos Colectivos y del Ambiente. 
De Jonge, M., Tipping, E., Lofts, S., Bervoets, L., \& Blust, R. (2013). The use of invertebrate body burdens to predict ecological effects of metal mixtures in mining-impacted waters. Aquatic Toxicology, 142143, 294-302.

Domínguez, E., Molineri, C., Pescador, M., Hubbard, M. D., \& Nieto, C. (2006). Aquatic Biodiversity in Latin America. Vol 2: Ephemeroptera of South America. Moscú: Pensoft.

Domínguez, E., \& Fernández, H. (2009). Macroinvertebrados bentónicos sudamericanos: Sistemática y biología. Tucumán: Fundación Miguel Lillo.

Edegbene, A. O., Arimoro, F. O., Odoh, O., \& Ogidiaka, E. (2015). Effect of anthropogenicity on the composition and diversity of aquatic insects of a municipal river in North Central Nigeria. Biosciences Research in Today's World, 1(1), 55-66.

Etter, A., McAlpine, C., Wilson, K., Phinn, S., \& Possingham, H. (2006). Regional patterns of agricultural land use and deforestation in Colombia. Agriculture, Ecosystems and Environment, 114, 369-386.

Fahrig, L. (2003). Effects of Habitat Fragmentation on Biodiversity. Annual Review of Ecology, Evolution and Systematics, 34, 487-515.

Feminella, J. W. (1996). Comparision of benthic macroinvertebrates assemblages in small streams along a gradient of flow permanence. Journal of the North American Benthological Society, 15, 651-669.

Ferreira da Silva, E., Almeida, S., Nunes, M., Luís, A., Borg, F., Hedlund, M., Marques de Sá, C., ..., \& Teixeira, P. (2009). Heavy metal pollution downstream the abandoned Coval da Mó mine (Portugal) and associated effects on epilithic diatom communities. Science of the Total Environment, 407, 5620-5636.

Giraldo, L. P., Chará, J., Zúñiga, M. C., Chará-Serna, A. M., \& Pedraza, G. (2014). Impacto del uso del suelo agropecuario sobre macroinvertebrados acuáticos en pequeñas quebradas de la cuenca del río La Vieja (Valle del Cauca, Colombia). Revista de Biología Tropical, 62(2), 203-219.

González, S. M., Ramírez, Y. P., Meza-S, A. M., \& G-Dias, L. (2012). Diversidad de macroinvertebrados acuáticos y calidad de agua de quebradas abastecedoras del municipio de Manizales. Boletín científico Museo de Historia Natural, 16(2), 135-148.

Greenacre, M. J. (1984). Theory and Applications of Correspondence Analysis. London: Academic Press.

Guevara, G., Godoy, R., Boeckx, P., Jara, C., \& Oyarzún, C. E. (2009). Leaf litter dynamics in headwater streams of the Chilean Andes: influence of shredders and silvicultural activities. In C. E. Oyarzún, N E. C. Verhoest, P. Boeckx, \& R. Godoy (Eds.),
Ecological advances on Chilean temperate rainforests (pp. 51-54). Ghent: Academia Press.

Gutiérrez, Y. \& G-Dias, L. (2015). Ephemeroptera (Insecta) de Caldas - Colombia, claves taxonómicas para los géneros y notas sobre su distribución. Museu de Zoologia da Universidade da São Paulo, 55(2), 13-46.

Harvey, C. A., Komar, O., Chazdon, R., Ferguson, B. G., Finegan, B., Griffith, D. M., Matínez-Ramos, ..., \& Wishnie, M. (2008). Integrating agricultural landscapes with biodiversity conservation in the Mesoamerican hotspot. Conservation Biology, 22(1), 8-15.

Hepp, L. U., Milesi, S. V., Biasi, C., \& Restello, R. M. (2010). Effects of agricultural and urban impacts on macroinvertebrates assemblages in streams (Rio Grande do Sul, Brazil). Zoologia, 27(1), 106-113.

Hershey, A. E., \& Lamberti, G. A. (1998). Stream macroinvertebrate communities. In R. J. Naiman \& R. E. Bilby (Eds.), River Ecology and ManagementLessons from the Pacific coastal ecoregion (pp: 169199). New York: Springer-Verlag.

Holdridge, L. R. (1982). Ecología basada en Zonas de Vida. Costa Rica: Instituto Interamericano de Cooperación para la Agricultura (IICA).

Humphries, S. (2002). Dispersal in drift-prone macroinvertebrates: a case for density-independence. Freshwater Biology, 47, 921-929.

Huston, M. A. (1994). Biological diversity: the coexistence of species on changing landscapes. Cambridge: Cambridge University Press.

Jergentz, S., Mugni, H., Bonetto, C., \& Schulz, R. (2004). Runoff-related endosulfan contamination and aquatic macroinvertebrate response in rural basins near Buenos Aires, Argentina. Archives of Environmental Contamination and Toxicology, 46, 345-352.

Jonsson, M., Malmqvist, B., \& Hoffsten, P. E. (2001). Leaf litter breakdown rates in boreal streams: does shredder species richness matter? Freshwater Biology, 46, 161-171.

Laabs, V. W., Amelung, A. A., Pinto, M., Wantzen, K. M., da Silva, C. J., \& Zech, W. (2002). Pesticides in surface water, sediments and rainfall of the north-eastern Pantanal basin, Brazil. Journal of Environmental Quality, 31, 1636-1648.

Lal, R. (1998). Effects of macrofauna on soil properties in tropical ecosystems. Agriculture Ecosystems \& Environment, 24, 101-106.

Longo, M., Zamora, H., Guisande, C., \& Ramírez, J. J. (2010). Dinámica de la comunidad de macroinvertebrados en la quebrada Potrerillos (Colombia): Respuesta a los cambios estacionales de caudal. Limnetica, 29(2), 195-210. 
McArdle, B., \& Anderson, M. (2001). Fitting multivariate models to semimetric distances: A comment on distance-based redundancy analysis. Ecology, 82, 290-297.

Mackey, R. L., \& Currie, D. J. (2001). The Diversity disturbance relationship: is it generally strong and peaked? Ecology, 82, 3479-3492.

Marrugo-Negrete, J. L., \& Paternina-Uribe, R. (2011). Evaluación de la contaminación por metales pesados en la ciénaga La Soledad y Bahía de Cispatá, cuenca de Cispatá cuenca del Bajo Sinú. Universidad de Córdoba, Córdoba, España.

Maloney, K. O., \& Weller, D. E. (2011). Anthropogenic disturbance and streams: land use and land-use change affect stream ecosystems via multiple pathways. Freshwater Biology, 56, 611-626.

Merritt, R. W., Cummins, K. W., \& Berg, M. B. (2008). An Introduction to the Aquatic Insects of North America. Fourth edition. Dubuque: Kendall /Hunt Publishing.

Meza-S, A. M., Rubio-M, J., G-Dias, L., \& Walteros, J. M. (2012). Calidad de agua y composición de macroinvertebrados acuáticos en la subcuenca alta del río Chinchiná. Caldasia, 34(2), 443-456.

Milesi, S. V., Biasi, C., Restello, R. M., \& Hepp, L. U. (2008). Efeito de metais Cobre $(\mathrm{Cu})$ e Zinco $(\mathrm{Zn})$ sobre a comunidade de macroinvertebrados bentônicos em riachos do sul do Brasil. Acta Scientiarum - Biological Science, 30(3), 283-289.

Mondal, K., \& Jana, S. (2015). A review of the effects of heavy metals on the aquatic animal of three different districts of West Bengal. Journal of Global Biosciences, 4(6), 2504-2512.

Moreno, C. A., Barragán, F., Pineda, E., \& Pavón, N. P. (2011). Reanálisis de la diversidad alfa: alternativas para interpretar y comparar información sobre comunidades ecológicas. Revista Mexicana de Biodiversidad, 84, 1249-1261.

Naiman, R. J., Décamps, H., \& McClain, M. E. (2005). Riparia: Ecology, conservation, and management of stream side communities. Burlington: Elsevier.

Nessimian, J. L., Venticinque, E. M., Zuanon, J., Marco-Jr, P., Gordo, M., Fidelis, L., Batista, J. D., \& Juen, L. (2008). Land use, habitat integrity, and aquatic insect assemblages in Central Amazonian streams. Hydrobiologia, 614(1), 117-131.

Newbold, T., Hudson, L. N., Hill, S. L. L., Contu, S., Lysenko, I., Senior, R. A., Börger, L., ..., \& Purvis, A. (2015). Global effects of land use on local terrestrial biodiversity. Nature, http://doi.org/10.1038/ nature 14324
Nicholls, C. I. (2008). Control biológico de insectos: un enfoque agroecológico. Medellín: Universidad de Antioquia.

Nilsson, C., Reidy, C. A., Dynesius, M., \& Revenga, C. (2005). Fragmentation and flow regulation of the world's large river systems. Science, 308, 405-408.

Palmer, M. A., Bernhardt, E. S., Schlesinger, W. H. Eshleman, K. N., Foufoula-Georgiou, E., Hendryx, M. S., Lemly, A. D., ..., \& Wilcock, P. R. (2010). Mountaintop Mining Consequences. Science, 327, 148-149.

Piggott, J. J., Lange, K., Townsend, C. T., \& Matthaei, C. D. (2012). Multiple stressors in agricultural streams: a mesocosm study of interactions among raised water temperature, sediment addition and nutrient enrichment. PloS ONE, 7, 1-14.

Pond, G. J., Passmore, M. E., Borsuk, F. A., Reynolds, L., \& Rose, C. J. (2008). Downstream effects of mountaintop coal mining: comparing biological conditions using family- and genus-level macroinvertebrate Bioassessment tools. Journal of the North American Benthological Society, 27(3), 717-737.

Ramírez, A., \& Viña, G. (1998). Limnología colombiana: Aportes a su conocimiento y estadísticas de análisis. Bogotá: Fundación Universidad de Bogotá Jorge Tadeo Lozano.

Ramírez, A., Pringle, C. M., \& Wantzen, K. M. (2008). Tropical stream conservation. In D. Dudgeon (Ed.), Tropical stream ecology (pp. 285-300). Burlington: Academic Press.

Reece, P., \& Richardson, J. S. (2000). Biomonitoring with the reference condition approach for the detection of aquatic ecosystem at risk. In L. M. Dearling (Ed.), Proceedings of a conference on the biology and management of species and habitats at risk (pp: 549552). Kamloops, B.C.

R Core Team. (2015). R: a language and environment for statistical computing. Vienna: $\mathrm{R}$ foundation for Statistical Computing. Retrieved from http://www.Rproject.org/

Riseng, C. M., Wiley, M. J., Black, R. W., \& Munn, M. D. (2011). Impacts of agricultural land use on biological integrity: a causal analysis. Ecological Applications, $21,3128-3146$.

Rodríguez-Barrios, J., Ospina-Torres, R., Gutiérrez, J., \& Ovalle, E. (2007). Densidad y biomasa de macroinvertebrados acuáticos derivantes en una quebrada tropical de montaña, Bogotá, Colombia. Caldasia, 29(2), 397-412.

Rodríguez-Barrios, J., Ospina-Tórres, R., \& Turizo-Correa, R. (2011). Grupos funcionales alimentarios de macroinvertebrados acuáticos en el río Gaira, Colombia. Revista de Biología Tropical, 54(9), 1537-1552. 
Roldán, G. (1996). Guía para el estudio de los macroinvertebrados acuáticos del Departamento de Antioquía. Medellín: Colciencias - Universidad de Antioquia.

Seoane, J. (2014). ¿Modelos mixtos (lineales)? Una introducción para el usuario temeroso. Etología, 24, 15-37.

Smith, H., Wood, P. J., \& Gunn, J. (2003). The influence of habitat structure and flow permanence on invertebrate communities in karst spring systems. Hydrobiologia, $510,53-66$

Smith, R. F., \& Lamp, W. O. (2008). Comparison of insect communities between adjacent headwater and main-stem streams in urban and rural watersheds. Journal of the North American Benthological Society, 27(1), 161-175.

Stanford, J. A., Ward, J. V., Liss, W. J., Frissel, C. A., Williams, R. N., Lichatowich, J. A., \& Coutant, C. C. (1996). A general protocol for restoration of regulated rivers. Research and Management, 12, 391-413.

Stanley, E. H., \& Fisher, S. G. (1992). Intermittency, Disturbance, and Stability in Stream Ecosystems. In R. D. Robarts \& M. L. Bothwell (Eds.), Aquatic Ecosystems in Semiarid Regions: Implications for Resource Management (pp: 271-280). Saskatoon: Environmental Canada.

StatSoft, Inc. (2007). STATISTICA (data analysis software system), version 8.0. Retrieved from http://www. statsoft.com

Subramanian, K. A., Sivaramakrishnan, K. G., \& Gadgil, M. (2005). Impact of riparian land use on stream insects of Kudremukh National Park, Karnataka state, India. Journal of Insect Science, 5(49), 1-10.

Tamaris-Turizo, C., Rodríguez-barrios, J., \& Ospina, R. (2013). Deriva de macroinvertebrados acuáticos a lo largo del río Gaira, vertiente noroccidental de la sierra nevada de Santa Marta, Colombia. Caldasia, 35(1), 149-163.

Ter Braak, C. J. F., \& Šmilauer, P. (2002). CANOCO Reference manual and CanoDraw for Windows user's guide: software for Canonical Community Ordination (version 4.5). Ithaca: Micro Power Ithaca.

Torres, P. J., \& Ramírez, A. (2014). Land use effects on leaf litter breakdown in low-order streams draining a rapidly developing tropical watershed in Puerto Rico. Revista de Biología Tropical, 62(2), 129-142.

Vannote, R. L., Minshall, G. W., Cummins, K. W., Sedell, K. R., \& Cushing, C. E. (1980). The river continuum concept. Canadian Journal of Fisheries and Aquatic Sciences, 37, 130-137.

Ward, J. V., Tockner, K., \& Schiemer, F. (1999). Biodiversity of floodplain river ecosystems: ecotones and connectivity. Research and Management, 15, 125-139.

Wiens, J. A. (2002). Riverine landscapes: taking landscape ecology into the water. Freshwater Biology, 47(4), 501-51.

Wood, P. J., \& Armitage, P. D. (1997). Biological effects of fine sediment in the lotic environment. Environmental Management, 21, 203-217.

Yoshimura, M. (2012). Effects of forest disturbances on aquatic insect assemblages. Entomological Science, $15,145-154$.

Zúñiga, M. C., \& Cardona, W. (2009). Bioindicadores de calidad de agua y caudal ambiental. In J. Cantera, Y. Carvajal, \& L. M. Castro (Eds.), Caudal Ambiental: Conceptos, Experiencias y Desafios (pp. 167-196). Cali: Programa Editorial Universidad del Valle. 


\section{ANEXO 1}

Características físicas, químicas e hidrológicas de las quebradas evaluadas

\section{APPENDIX 1}

Physical, chemical, and hydrological characteristics of the evaluated streams

\begin{tabular}{|c|c|c|c|c|c|c|c|c|}
\hline \multirow{2}{*}{ Parámetro } & \multirow{2}{*}{ Cod } & \multirow{2}{*}{ Unidades } & \multicolumn{6}{|c|}{ Estación } \\
\hline & & & E1 & E2 & E3 & E4 & E5 & E6 \\
\hline Temperatura del agua & $\mathrm{T}^{\circ}$ & ${ }^{\circ} \mathrm{C}$ & 13.25 & 13.38 & 14.38 & 18.90 & 18.48 & 19.70 \\
\hline $\mathrm{pH}$ & $\mathrm{pH}$ & - & 7.38 & 7.38 & 7.28 & 8.27 & 7.70 & 8.08 \\
\hline Conductividad & Con & $\mu \mathrm{s}$ & 209 & 86 & 83 & 100 & 35 & 84 \\
\hline $\mathrm{O}_{2}$ disuelto & $\mathrm{O}_{2}$ & $\mathrm{mg} / \mathrm{L}$ & 5.29 & 5.88 & 5.19 & 2.75 & 5.93 & 2.08 \\
\hline $\mathrm{DBO}_{5}$ & $\mathrm{DBO}_{5}$ & $\mathrm{mg} / \mathrm{L}$ & 3.21 & 6.17 & 3.21 & 3.21 & 3.21 & 3.21 \\
\hline DQO & DQO & $\mathrm{mg} / \mathrm{L}$ & 32.67 & 91.67 & 59.67 & 29.67 & 55.33 & 25.00 \\
\hline Coliformes totales & Col_T & $\mathrm{UFC} / 100 \mathrm{ml}$ & 1955 & 4050 & 2082 & 2200 & 6600 & 13350 \\
\hline Coliformes fecales & Col_F & $\mathrm{UFC} / 100 \mathrm{ml}$ & 755 & 2300 & 771 & 493 & 2200 & 8450 \\
\hline Sólidos totales & ST & $\mathrm{mg} / \mathrm{L}$ & 85 & 569 & 161 & 124 & 311 & 113 \\
\hline Sólidos suspendidos totales & SST & $\mathrm{mg} / \mathrm{L}$ & 6 & 237 & 21 & 15 & 74 & 8 \\
\hline Cianuro & $\mathrm{CN}$ & $\mathrm{mg} / \mathrm{L}$ & 0.12 & 0.12 & 0.12 & 0.12 & 0.12 & 0.13 \\
\hline Boro & $\mathrm{B}$ & $\mathrm{mg} / \mathrm{L}$ & 1.15 & 1.15 & 1.15 & 1.15 & 1.15 & 1.15 \\
\hline Plomo & $\mathrm{Pb}$ & $\mathrm{mg} / \mathrm{L}$ & 0.02 & 0.02 & 0.02 & 0.02 & 0.02 & 0.02 \\
\hline Mercurio & $\mathrm{Hg}$ & $\mathrm{mg} / \mathrm{L}$ & 0.003 & 0.003 & 0.003 & 0.003 & 0.003 & 0.003 \\
\hline Nitrógeno amoniacal & $\mathrm{NH}_{3}-\mathrm{N}$ & $\mathrm{mg} / \mathrm{L}$ & 0.11 & 0.36 & 0.11 & 0.06 & 0.16 & 0.17 \\
\hline Fosfatos & $\mathrm{PO}_{4}$ & $\mathrm{mg} / \mathrm{L}$ & 0.30 & 2.33 & 0.20 & 0.30 & 0.43 & 0.23 \\
\hline Sulfatos & $\mathrm{SO}_{4}$ & $\mathrm{mg} / \mathrm{L}$ & 7.67 & 45.67 & 16.00 & 11.33 & 9.33 & 9.67 \\
\hline Hierro & $\mathrm{Fe}$ & $\mathrm{mg} / \mathrm{L}$ & 0.16 & 1.87 & 0.37 & 0.06 & 0.81 & 6.16 \\
\hline Cloruros & $\mathrm{Cl}-$ & $\mathrm{mg} / \mathrm{L}$ & 3.03 & 7.30 & 2.50 & 2.50 & 3.20 & 2.50 \\
\hline Nitratos & $\mathrm{NO}_{3}$ & $\mathrm{mg} / \mathrm{L}$ & 1.00 & 1.03 & 1.00 & 1.00 & 1.00 & 1.00 \\
\hline Nitritos & $\mathrm{NO}_{2}$ & $\mathrm{mg} / \mathrm{L}$ & 0.07 & 0.34 & 0.07 & 0.07 & 0.10 & 0.10 \\
\hline Aluminio & $\mathrm{Al}$ & $\mathrm{mg} / \mathrm{L}$ & 0.09 & 0.2 & 0.32 & 0.2 & 2.01 & 0.2 \\
\hline Grasas y aceites & GyA & $\mathrm{mg} / \mathrm{L}$ & 0.50 & 0.50 & 0.50 & 0.83 & 0.50 & 0.50 \\
\hline Profundidad promedio & Prof & $\mathrm{cm}$ & 17.27 & 22.01 & 13.49 & 7.59 & 7.03 & 6.42 \\
\hline Ancho & Anc & $\mathrm{m}$ & 6.28 & 4.67 & 5.12 & 1.82 & 1.26 & 1.01 \\
\hline Velocidad del cauce & Vel & $\mathrm{m} / \mathrm{s}$ & 0.71 & 0.66 & 0.50 & 0.38 & 0.18 & 0.37 \\
\hline Caudal & Q & $\mathrm{m}^{3} / \mathrm{s}$ & 0.77 & 0.71 & 0.38 & 0.09 & 0.02 & 0.04 \\
\hline Altitud & Alt & $\mathrm{m}$ & 2305 & 2260 & 2275 & 1720 & 1849 & 1754 \\
\hline
\end{tabular}

Los valores corresponden a los promedios de los parámetros medidos en cada estación de muestreo. E1: Quebrada Romerales (zona de referencia), E2: Quebrada California (minería 1), E3: Quebrada Toldafría (minería 2), E4: Quebrada El Establo (zona de referencia), E5: Quebrada Don Alonso (agricultura 1) y E6: Quebrada Don Fernando (agricultura 2). The values correspond to the averages of the parameters measured at each sampling station. E1: Romerales stream (reference zone), E2: California stream (mining 1), E3: Toldafría stream (mining 2), E4: El Establo stream (reference zone), E5: Don Alonso steam (agriculture 1) and E6: Don Fernando stream (agriculture 2). 
ANEXO 2

Listado de morfoespecies por estación de muestreo

APPENDIX 2

Morphospecies list for each sampling station. We show the abbreviation (Abrev) for each one

\begin{tabular}{|c|c|c|c|c|c|c|c|c|c|c|c|}
\hline \multirow{3}{*}{ Orden } & \multirow{3}{*}{ Familia } & \multirow{3}{*}{ Morfoespecie } & \multirow{3}{*}{ Abrev } & \multicolumn{6}{|c|}{ Localidad } & \multirow{3}{*}{ Total } & \multirow{3}{*}{$\mathrm{N} \%$} \\
\hline & & & & \multicolumn{3}{|c|}{ Gallinazo } & \multicolumn{3}{|c|}{ Llanitos } & & \\
\hline & & & & E1 & E2 & E3 & E4 & E5 & E6 & & \\
\hline \multirow[t]{18}{*}{ Coleoptera } & Cantharidae & Cantharidae sp. 1 & Can1 & 4 & 3 & 2 & 0 & 0 & 0 & 9 & 0.1 \\
\hline & Dryopidae & Dryopidae sp. 1 & Dry1 & 0 & 0 & 0 & 2 & 0 & 0 & 2 & 0.0 \\
\hline & Dytiscidae & Dytiscidae sp. 1 & Dys1 & 2 & 0 & 0 & 0 & 1 & 0 & 3 & 0.0 \\
\hline & \multirow[t]{7}{*}{ Elmidae } & Austrolimnius & Aus & 0 & 0 & 0 & 1 & 0 & 0 & 1 & 0.0 \\
\hline & & Cylloepus & Cyl & 10 & 2 & 10 & 16 & 1 & 0 & 39 & 0.3 \\
\hline & & Elmidae sp. 1 & Elm1 & 0 & 0 & 9 & 0 & 0 & 0 & 9 & 0.1 \\
\hline & & Heterelmis & Het & 4 & 16 & 12 & 13 & 0 & 0 & 45 & 0.4 \\
\hline & & Macrelmis & Mac & 51 & 26 & 6 & 10 & 0 & 0 & 93 & 0.7 \\
\hline & & Neoelmis & $\mathrm{Neo}$ & 28 & 4 & 10 & 0 & 0 & 0 & 42 & 0.3 \\
\hline & & Pharceonus & Pha & 0 & 1 & 0 & 0 & 0 & 0 & 1 & 0.0 \\
\hline & \multirow[t]{2}{*}{ Hydrophilidae } & Hydrophilidae sp. 1 & Hael & 0 & 0 & 4 & 0 & 0 & 39 & 43 & 0.3 \\
\hline & & Tropisternus & Tro & 0 & 1 & 0 & 0 & 0 & 0 & 1 & 0.0 \\
\hline & Nitidulidae & Nitidulidae sp. 1 & Nit & 0 & 0 & 1 & 0 & 0 & 0 & 1 & 0.0 \\
\hline & Psephenidae & Psephenus & Pse & 1 & 0 & 0 & 0 & 0 & 0 & 1 & 0.0 \\
\hline & Ptilodactylidae & Anchytarsus & Anc & 8 & 14 & 29 & 82 & 4 & 0 & 137 & 1.1 \\
\hline & Scirtidae & Scirtidae sp. 1 & Scir & 16 & 24 & 28 & 0 & 0 & 0 & 68 & 0.5 \\
\hline & \multirow[t]{2}{*}{ Staphylinidae } & Staphylinidae sp. 1 & Sta1 & 14 & 12 & 8 & 3 & 1 & 11 & 49 & 0.4 \\
\hline & & Staphylinidae sp. 2 & Sta2 & 3 & 2 & 2 & 0 & 1 & 6 & 14 & 0.1 \\
\hline \multirow[t]{26}{*}{ Diptera } & Blephariceridae & Limonicola & Lla & 43 & 4 & 2 & 0 & 0 & 0 & 49 & 0.4 \\
\hline & Ceratopogonidae & Bezzia & Bez & 15 & 2 & 2 & 0 & 0 & 4 & 23 & 0.2 \\
\hline & \multirow[t]{9}{*}{ Chironomidae } & Chironominae sp. 1 & Cael & 67 & 40 & 40 & 104 & 2 & 203 & 456 & 3.6 \\
\hline & & Chironominae sp. 2 & $\mathrm{Cae} 2$ & 1 & 0 & 0 & 0 & 0 & 0 & 1 & 0.0 \\
\hline & & Chironomini sp. 1 & Cnil & 0 & 3 & 0 & 0 & 0 & 0 & 3 & 0.0 \\
\hline & & Chironomus & Chi & 0 & 0 & 4 & 0 & 0 & 0 & 4 & 0.0 \\
\hline & & Cricotopus & Cus & 745 & 274 & 100 & 328 & 5 & 657 & 2109 & 16.8 \\
\hline & & Diamesinae sp. 1 & Dial & 1 & 0 & 0 & 0 & 0 & 0 & 1 & 0.0 \\
\hline & & Podonomus & Pod & 3 & 5 & 2 & 0 & 0 & 0 & 10 & 0.1 \\
\hline & & Tanypodinae sp. 1 & Tan1 & 74 & 9 & 4 & 53 & 1 & 11 & 152 & 1.2 \\
\hline & & Tanytarsini sp. 1 & Tnil & 1 & 0 & 0 & 164 & 0 & 0 & 165 & 1.3 \\
\hline & Dixidae & Dixidae sp. 1 & Dix & 2 & 0 & 0 & 1 & 0 & 0 & 3 & 0.0 \\
\hline & Dolichopodidae & Dolichopodidae sp. 1 & Doll & 0 & 0 & 0 & 2 & 0 & 0 & 2 & 0.0 \\
\hline & Empididae & Empididae sp. 1 & Emp1 & 7 & 15 & 0 & 5 & 0 & 3 & 30 & 0.2 \\
\hline & Muscidae & Muscidae sp. 1 & Mus1 & 13 & 3 & 14 & 2 & 0 & 22 & 54 & 0.4 \\
\hline & Psychodidae & Psychodidae sp. 1 & Psyl & 0 & 0 & 0 & 0 & 0 & 1 & 1 & 0.0 \\
\hline & \multirow[t]{2}{*}{ Simuliidae } & Gigantodax & Gig & 4 & 1 & 0 & 0 & 0 & 0 & 5 & 0.0 \\
\hline & & Simulium & Sim & 295 & 51 & 87 & 77 & 686 & 1423 & 2619 & 20.9 \\
\hline & \multirow[t]{8}{*}{ Tipulidae } & Hexatoma & Hex & 0 & 0 & 1 & 0 & 0 & 0 & 1 & 0.0 \\
\hline & & Limonia & Lim & 2 & 1 & 2 & 0 & 0 & 0 & 5 & 0.0 \\
\hline & & Molophilus & Mol & 6 & 15 & 6 & 8 & 1 & 0 & 36 & 0.3 \\
\hline & & Tipula & Tip & 8 & 15 & 21 & 25 & 13 & 7 & 89 & 0.7 \\
\hline & & Tipulidae sp. 1 & Tael & 3 & 0 & 1 & 3 & 0 & 19 & 26 & 0.2 \\
\hline & & Tipulidae sp. 2 & Tae2 & 1 & 1 & 0 & 1 & 0 & 0 & 3 & 0.0 \\
\hline & & Diptera sp. 1 & Dip1 & 0 & 0 & 1 & 0 & 0 & 1 & 2 & 0.0 \\
\hline & & Diptera sp. 2 & Dip2 & 0 & 0 & 1 & 0 & 0 & 0 & 1 & 0.0 \\
\hline Ephemeroptera & Baetidae & Andesiops & And & 393 & 167 & 215 & 46 & 0 & 1 & 822 & 6.6 \\
\hline & & Baetodes & $\mathrm{Bae}$ & 679 & 906 & 451 & 458 & 1 & 1 & 2496 & 19.9 \\
\hline & & Camelobaetidius & Cam & 327 & 70 & 4 & 2 & 0 & 0 & 403 & 3.2 \\
\hline & & Mayobaetis & May & 11 & 3 & 8 & 6 & 0 & 0 & 28 & 0.2 \\
\hline & & Nanomis & Nan & 73 & 19 & 195 & 43 & 0 & 0 & 330 & 2.6 \\
\hline & & Paracloeodes & Par & 1 & 0 & 6 & 5 & 0 & 0 & 12 & 0.1 \\
\hline
\end{tabular}


APÉNDICE 2 (Continuación) / APPENDIX 2 (Continued)

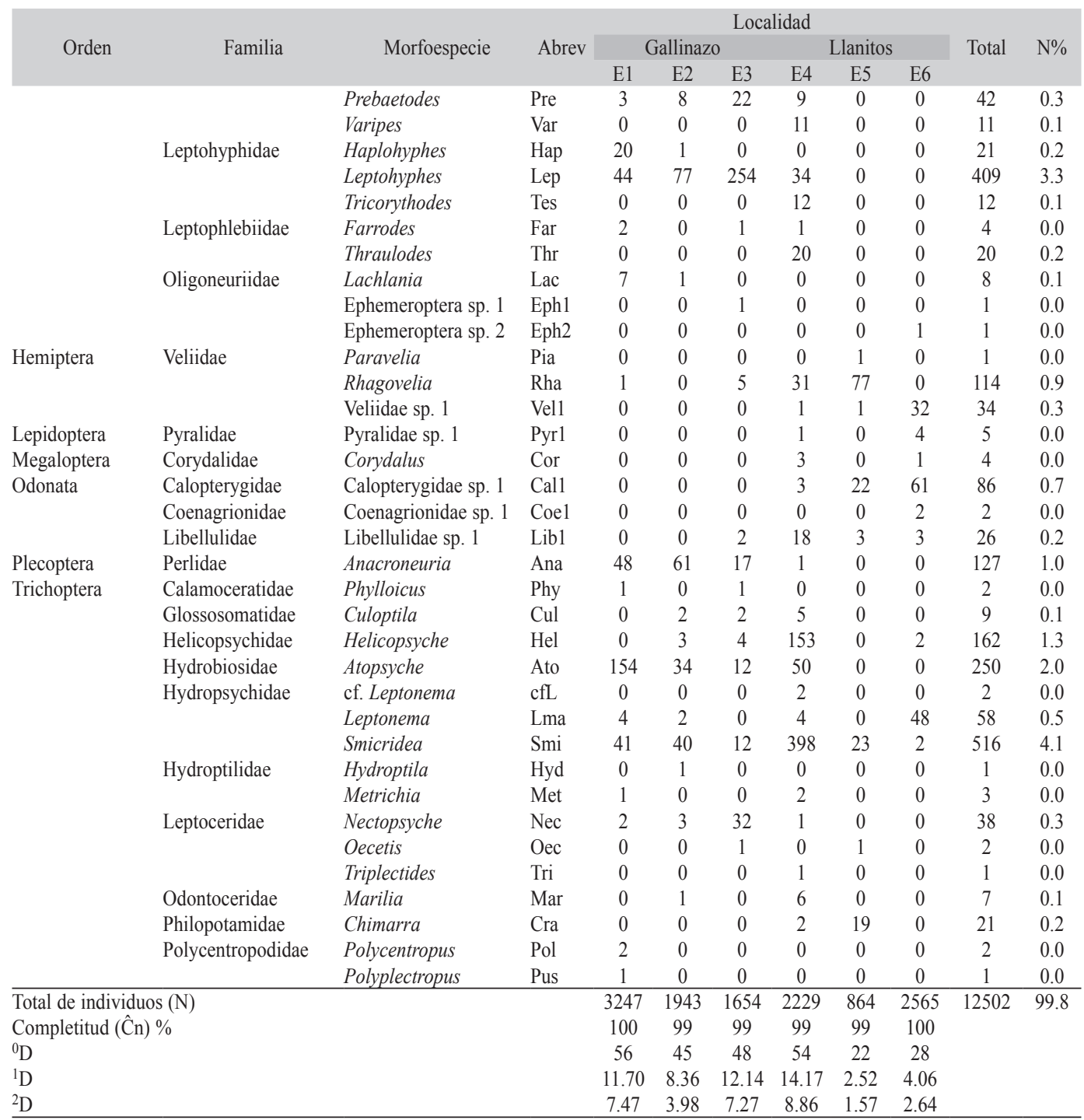

Se presenta la abreviación (Abrev) para cada uno. La última columna muestra la abundancia proporcional de cada taxa con respecto al total de individuos.

The last column shows the proportional abundance of each taxa with respect to the total of individuals.

$\mathrm{N}=$ abundancia.

$\% \mathrm{~N}=$ abundancia porcentual por taxón.

$\hat{\mathrm{C}} \mathrm{n}=$ completitud de muestreo.

${ }^{0} \mathrm{D}=$ riqueza.

${ }^{1} \mathrm{D}=$ diversidad con Shannon.

${ }^{2} \mathrm{D}=$ diversidad con Simpson. 


\section{ANEXO 3}

Resultados del análisis de porcentaje de similitud (SIMPER) para los factores impacto y sustrato

\section{APPENDIX 3}

Results of the similarity percentage analysis (SIMPER) for impact and substrate factors

\begin{tabular}{|c|c|c|c|c|}
\hline \multicolumn{5}{|c|}{ Comparaciones por tipo de impacto } \\
\hline \multirow{2}{*}{ Morfoespecie } & \multicolumn{3}{|c|}{ Abundancia promedio $(\%)$} & \multirow{2}{*}{$\mathrm{P}$ valor } \\
\hline & Minería & Referencia & Contribución acumulativa & \\
\hline Baetodes & 13.165049 & 10.527778 & 0.217 & 0.01 \\
\hline Cricotopus & 3.631068 & 9.935185 & 0.371 & 0.43 \\
\hline Andesiops & 3.708738 & 4.064815 & 0.4551 & 0.01 \\
\hline Smicridea & 0.504854 & 4.064815 & 0.5201 & 0.01 \\
\hline Simulium & 1.339806 & 3.444444 & 0.5724 & 1 \\
\hline Camelobaetidius & 0.718447 & 3.046296 & 0.6206 & 0.01 \\
\hline Nanomis & 2.019417 & 1.074074 & 0.6668 & 0.01 \\
\hline Leptohyphes & 3.213592 & 0.722222 & 0.7071 & 0.01 \\
\hline Chironominae sp. 1 & 0.776699 & 1.583333 & 0.7438 & 1 \\
\hline Atopsyche & 0.446602 & 1.888889 & 0.7722 & 0.01 \\
\hline Helicopsyche & 0.07767 & 1.416667 & 0.7946 & 0.01 \\
\hline Tanypodinae sp. 1 & 0.126214 & 1.175926 & 0.8144 & 0.01 \\
\hline Anchytarsus & 0.417476 & 0.833333 & 0.834 & 0.01 \\
\hline Anacroneuria & 0.757282 & 0.453704 & 0.8503 & 0.03 \\
\hline \multirow{2}{*}{ Morfoespecie } & \multicolumn{3}{|c|}{ Abundancia promedio (\%) } & \multirow{2}{*}{$\mathrm{P}$ valor } \\
\hline & Referencia & Agricultura & Contribución acumulativa & \\
\hline Simulium & 3.444444 & 21.30303 & 0.2108 & 0.01 \\
\hline Cricotopus & 9.935185 & 6.68687 & 0.3813 & 0.01 \\
\hline Baetodes & 10.527778 & 0.0202 & 0.5011 & 1 \\
\hline Smicridea & 4.064815 & 0.25253 & 0.5641 & 0.01 \\
\hline Andesiops & 4.064815 & 0.0101 & 0.6223 & 0.94 \\
\hline Chironominae sp. 1 & 1.583333 & 2.07071 & 0.6701 & 0.18 \\
\hline Camelobaetidius & 3.046296 & 0 & 0.7099 & 0.01 \\
\hline Atopsyche & 1.888889 & 0 & 0.7343 & 0.01 \\
\hline Rhagovelia & 0.296296 & 0.77778 & 0.7564 & 0.25 \\
\hline Helicopsyche & 1.416667 & 0.0202 & 0.7775 & 0.01 \\
\hline Nanomis & 1.074074 & 0 & 0.7965 & 1 \\
\hline Tanypodinae sp. 1 & 1.175926 & 0.12121 & 0.8155 & 0.01 \\
\hline Calopterygidae sp. 1 & 0.027778 & 0.83838 & 0.8327 & 0.08 \\
\hline Anchytarsus & 0.833333 & 0.0404 & 0.8486 & 0.14 \\
\hline
\end{tabular}




\begin{tabular}{|c|c|c|c|c|}
\hline \multirow{2}{*}{ Morfoespecie } & \multicolumn{3}{|c|}{ Abundancia promedio $(\%)$} & \multirow{2}{*}{$\mathrm{P}$ valor } \\
\hline & Minería & Agricultura & Contribución acumulativa & \\
\hline Simulium & 1.339806 & 21.30303 & 0.2479 & 0.01 \\
\hline Baetodes & 13.165049 & 0.0202 & 0.4371 & 0.02 \\
\hline Cricotopus & 3.631068 & 6.68687 & 0.5533 & 0.95 \\
\hline Andesiops & 3.708738 & 0.0101 & 0.6232 & 0.03 \\
\hline Chironominae sp. 1 & 0.776699 & 2.07071 & 0.6718 & 0.08 \\
\hline Nanomis & 2.019417 & 0 & 0.7119 & 0.04 \\
\hline Leptohyphes & 3.213592 & 0 & 0.7493 & 0.01 \\
\hline Rhagovelia & 0.048544 & 0.77778 & 0.7757 & 0.02 \\
\hline Calopterygidae sp. 1 & 0 & 0.83838 & 0.7986 & 0.01 \\
\hline Smicridea & 0.504854 & 0.25253 & 0.8168 & 1 \\
\hline Anacroneuria & 0.757282 & 0 & 0.8324 & 0.01 \\
\hline Tipula & 0.349515 & 0.20202 & 0.8461 & 0.07 \\
\hline Camelobaetidius & 0.718447 & 0 & 0.8588 & 1 \\
\hline \multicolumn{5}{|c|}{ Comparaciones por tipo de sustrato } \\
\hline \multirow{2}{*}{ Morfoespecie } & \multicolumn{3}{|c|}{ Abundancia promedio (\%) } & \multirow{2}{*}{$\mathrm{P}$ valor } \\
\hline & Sedimento & Roca & Contribución acumulativa & \\
\hline Baetodes & 2.125 & 15.555556 & 0.224 & 0.01 \\
\hline Simulium & 1.42708 & 9.277778 & 0.3774 & 0.96 \\
\hline Cricotopus & 3.69792 & 5.851852 & 0.5184 & 0.58 \\
\hline Andesiops & 1.52083 & 2.675926 & 0.5892 & 0.17 \\
\hline Chironominae sp. 1 & 1.35417 & 1.148148 & 0.6377 & 0.21 \\
\hline Nanomis & 1.27083 & 1.212963 & 0.682 & 0.01 \\
\hline Smicridea & 0.25 & 1.796296 & 0.7223 & 0.88 \\
\hline Camelobaetidius & 0.8125 & 1.833333 & 0.762 & 0.02 \\
\hline Rhagovelia & 0.36458 & 0.435185 & 0.7852 & 0.32 \\
\hline Atopsyche & 0.125 & 0.851852 & 0.8024 & 0.95 \\
\hline Tanypodinae sp. 1 & 0.6875 & 0.444444 & 0.8196 & 0.09 \\
\hline Helicopsyche & 0.46875 & 0.62963 & 0.8364 & 0.24 \\
\hline Calopterygidae sp. 1 & 0.13542 & 0.268519 & 0.8494 & 0.7 \\
\hline \multirow{2}{*}{ Morfoespecie } & \multicolumn{3}{|c|}{ Abundancia promedio $(\%)$} & \multirow{2}{*}{$\mathrm{P}$ valor } \\
\hline & Sedimento & Hojarasca & Contribución acumulativa & \\
\hline Simulium & 1.42708 & 13.962264 & 0.1837 & 0.11 \\
\hline Cricotopus & 3.69792 & 10.584906 & 0.3389 & 0.12 \\
\hline Baetodes & 2.125 & 5.764151 & 0.4472 & 1 \\
\hline Andesiops & 1.52083 & 3.650943 & 0.5147 & 0.43 \\
\hline Smicridea & 0.25 & 2.811321 & 0.5662 & 0.11 \\
\hline Chironominae sp. 1 & 1.35417 & 1.90566 & 0.6155 & 0.21 \\
\hline Leptohyphes & 0.0625 & 3.367925 & 0.6597 & 0.01 \\
\hline Nanomis & 1.27083 & 0.669811 & 0.6922 & 0.73 \\
\hline Camelobaetidius & 0.8125 & 1.198113 & 0.7188 & 0.89 \\
\hline Tipula & 0.0625 & 0.707547 & 0.7404 & 0.01 \\
\hline Atopsyche & 0.125 & 1.377358 & 0.7611 & 0.21 \\
\hline Rhagovelia & 0.36458 & 0.301887 & 0.78 & 0.49 \\
\hline Calopterygidae sp. 1 & 0.13542 & 0.415094 & 0.7972 & 0.09 \\
\hline Anchytarsus & 0.05208 & 0.811321 & 0.814 & 0.11 \\
\hline Anacroneuria & 0.05208 & 0.858491 & 0.8304 & 0.03 \\
\hline Tanypodinae sp. 1 & 0.6875 & 0.358491 & 0.8447 & 0.49 \\
\hline Helicopsyche & 0.46875 & 0.471698 & 0.8582 & 0.69 \\
\hline
\end{tabular}




\begin{tabular}{lcccc}
\multicolumn{1}{r}{ Morfoespecie } & \multicolumn{3}{c}{ Abundancia promedio (\%) } & P valor \\
Simulium & Roca & Hojarasca & Contribución acumulativa & 0.1 \\
Baetodes & 9.277778 & 13.962264 & 0.1929 & 0.24 \\
Cricotopus & 15.555556 & 5.764151 & 0.3761 & 0.93 \\
Andesiops & 5.851852 & 10.584906 & 0.5112 & 0.98 \\
Smicridea & 2.675926 & 3.650943 & 0.5713 & 0.17 \\
Leptohyphes & 1.796296 & 2.811321 & 0.6248 & 0.01 \\
Chironominae sp. 1 & 0.425926 & 3.367925 & 0.6634 & 1 \\
Camelobaetidius & 1.148148 & 1.90566 & 0.7276 & 0.87 \\
Nanomis & 1.833333 & 1.198113 & 0.7516 & 0.98 \\
Atopsyche & 1.212963 & 0.669811 & 0.7742 & 0.12 \\
Rhagovelia & 0.851852 & 1.377358 & 0.7914 & 0.68 \\
Anchytarsus & 0.435185 & 0.301887 & 0.8082 & 0.23 \\
Anacroneuria & 0.425926 & 0.811321 & 0.8232 & 0.16 \\
Tipula & 0.287037 & 0.858491 & 0.8378 & 0.09 \\
Calopterygidae sp. 1 & 0.074074 & 0.707547 & 0.8515 & 0.66 \\
\hline
\end{tabular}

Se presentan las comparaciones al interior de cada factor.

Resultado general para estación: $\mathrm{R}=0.3258, \mathrm{P}$ valor $=0.001$; resultado general para sustrato: $\mathrm{R}=0.0818, \mathrm{P}$ valor $=0.001$. The comparisons are presented within each factor. General result for station: $\mathrm{R}=0.3258, \mathrm{P}$ value $=0.001$; general result for substrate: $\mathrm{R}=0.0818, \mathrm{P}$ value $=0.001$. 